\title{
Financing Human Capital Development By Increasing The Minimum Wage: Evidence From Canada
}

\author{
Mahmoud Yousef Askari, Al Ain University of Science and Technology, UAE
}

\begin{abstract}
This study provides empirical evidence that using the minimum wage as a tool to generate extra taxes to establish a fully publically-funded higher education system is a harmless approach to boost funding for human capital development without changing governments' spending priorities or raising current tax rates. The paper proposes a method to finance human capital development through higher education by generating more income taxes from a higher minimum wage and through an effective link of the minimum wage to the Consumer Price Index (CPI) in Canada. The paper also argues that indexed minimum wage adjustments will help in fighting poverty, maintain an acceptable living standard for minimum wage workers, reduce dependence on government subsidies, and make-work more attractive. The paper concludes that using minimum wage adjustments as a tool to generate tax revenues and fund higher education could be an effective fiscal tool and could be considered a 'safe political instrument'.
\end{abstract}

Keywords: Human Capital Development; Minimum Wage; Canada

\section{INTRODUCTION}

1 $\mathrm{t}$ could be argued that investing in human capital is a necessity for all nations. Regardless of a nation's wealth of financial and natural resources, maintaining the intangible wealth of knowledge, skills, and capabilities of citizens could be inevitable. A major challenge in this process for many countries is the associated cost of education and training at all levels (K-12 \& post-secondary education) and the increasing number of learners in a knowledge-based global economy (Woodhall, 2007). Also, while many developing and developed nations are striving to properly fund a public K-12 education system, many, including Canada, are reducing public funding for higher education and gradually pushing public academic institutions towards a market driven, managed, and financed higher education system (Askari, 2014; AUCC, 2008; Metcalfe, 2010).

\section{OVERVIEW OF THE PROBLEM}

Unlike the sensitivity and attractiveness of healthcare and K-12 funding in Canada, higher education funding lacks the appeal to become an election campaign issue for political parties. Many politicians argue that more spending (for higher education) would have to be financed by higher taxes that could scare voters away from political parties. Consequently, many Liberal and Conservative policy makers have adopted Milton Friedman's approach to higher education funding who suggested that "it is eminently desirable that every young man and woman, regardless of his or her parents' income, social position, residence or race, have the opportunity to get higher education - provided that he or she is willing to pay for it." (Friedman \& Friedman, 1980, p. 183). This ideology has led to the use of tuition fees and student loans as funding alternatives and has led to the adoption of many business tools by many publicly funded academic institutions. Also, this has led many public universities in Canada to operate in what might be called: 'A Semi-Private Higher Education Industry'. Certainly, public academic institutions in Canada are still 'public' in terms of government influence and also due to the existing, but declining, government financial support for these institutions. At the same time, the private portion of the operating budgets of 
these institutions is growing, and tuition fees from both domestic and international students are becoming major sources of revenue (Hauptman \& Nolan, 2011; Sun \& Barrientos, 2009).

This paper proposes a sustainable solution to the problem of public funding for human capital development through higher education in Canada. The paper provides empirical evidence that provincial governments in Canada have other options to pay for higher education without the need to increase taxes, redesign their spending priorities, and, arguably, without suffering from heavy political consequences. The paper is an examination of Askari's (2014) suggested idea that adjusting the minimum wage could be a tool to generate enough tax revenues to fully fund public academic institutions. Using the minimum wage as a tool to pay for higher education is a harmless mechanism of funding that could give a 'win win' result for governments, learners, and minimum wage workers. In fact, "the political appeal for minimum wages as an anti-poverty device is obvious. Raising the wages of the working poor should enhance their earned income and reduce their likelihood of being in poverty" (Campolieti, Gunderson, \& Lee, 2012, p. 288). Dickson and Mayatt (2002) also stated that in Canada, minimum wage adjustments are used to enhance support for politicians to win them votes. Thus, using the minimum wage as a tool could be considered a 'safe political instrument'.

This study has analyzed a one-dollar difference in minimum wage and its effect on the generated life-long income tax revenues in the 13 Canadian provinces and territories. Unlike the United States that has federal, state, and, in some cases, local minimum wage legislations, Canada's minimum wage is under provincial jurisdiction and is legislated by provincial governments only. At the same time, each province has its own income tax rates to accompany the federal income tax rates (Appendix B \& C). This makes it easier for provincial governments to reevaluate their own minimum wage and income tax legislations and craft their own appropriate policies.

\section{THE MINIMUM WAGE ARGUMENT}

This paper assumes that firms have enough power to prevent the invisible hands of the market to determine a fair price (wage) in the labour market and assumes that firms are able to set prices below a 'just' market price for labour. In other words, the labour market is assumed to be in a 'Monopsony state', where buyers of labour services have a high degree of bargaining power and could easily control wages. Based on this logic, governments are obligated to interfere in setting wage rates that could be closer to a true market rate in a competitive labour market. According to Edward and Gilman (1999), some researchers (Manning, 1996) stated that Monopsony is a fact of life in all labour markets, and one need not to have the classical case of a company town to declare the existence of Monopsony. Governments' interference in mandating a minimum wage in the Monopsony case is similar to the interference of governments to protect consumers in Monopoly markets from higher Monopolist-determined prices.

This paper also assumes that indexed minimum wage adjustments will help in fighting poverty, maintain an acceptable living standard for minimum wage workers, reduce dependence on government subsidies, and makework more attractive. In fact, supporters of minimum wage legislations, according to Even and Macpherson (1996) highlight the "equalizing effect on income distribution and argue that increasing the minimum wage reduces poverty" (p. 67). Evan and Macpherson also added that those who are against minimum wage usually use the excuse of higher unemployment due to higher minimum wage. A study by Partridge and Partridge (1999) concluded that higher minimum wage would reduce employment and negatively affect poverty. Also, Campolieti, Fung, and Gunderson (2005) have concluded, "minimum wage increases led to large and statistically significant reductions in the employment of teenagers" (p. 533). Another Canadian study by Campolieti, Gunderson, and Lee (2012) also argued that a minimum wage increase in Canada would not reduce poverty because many working teenagers are coming from rich families.

Indeed, the argument that increasing the minimum wage will negatively affect youth employment might not have enough support in literature. According to Card and Krueger (1995), time series studies have shown an average of one percent decrease in youth employment due to a 10 percent increase in minimum wage, but recent studies "have estimated negligible or even marginally positive employment effect of the minimum wage" (p. 238). Card and Krueger further added that both researchers and academic journals might be guided by predetermined assumptions that higher minimum wage would decrease employment and they usually choose certain variables and samples to generate statistically significant results. Higher minimum wage, according to Card and Krueger, has the 
potential to increase employment because it could draw more workers to the labour market. They also concluded that there is a publication bias in the minimum wage published literature and stated "studies in the literature have been affected by specification-searching and publication biases, induced by editors' and authors' tendencies to look for negative and statistically significant estimates of the employment effect of the minimum wage" (p. 242).

Indexing the minimum wage to CPI is based on the logical argument that the minimum wage is assumed to be a living wage. But due to inflation, the real value of the wage will decrease, thus justifying an adjustment guided by an inflation index to maintain living standard. According to Even and Macpherson (1996), in the US, "the decline in the minimum's real value accounted for 20 to $30 \%$ of the rise in wage inequality during the $1980 \mathrm{~s} .$. . [and that indexing could be based on] consumer price index (CPI) and the average wage of non-supervisory private workers." (p. 68). Even and Macpherson added that using CPI as an index will help in maintaining the same living standard for minimum wage workers.

\section{RESEARCH METHODOLOGY}

To see the effect of a slight increase in personal earnings on the lifetime contributions of income taxes, two hypothetical citizens with a one dollar per hour difference in earnings (one makes $\$ 10.25 /$ hour and one makes $\$ 11.25$ / hour) were compared in every province and territory in Canada. Using the average tax rates in 2012 and Ernst and Young LLP public personal income tax calculator, lifetime annual income tax payments were calculated and compared with government transfer payment per person for $2012(\$ 6,122)$ that was calculated in Askari $(2014)$. Lifetime income tax payments to government for a citizen starting at minimum wage of $\$ 10.25 /$ hour with a two percent annual wage increase, and lifetime income tax payments for a citizen with one dollar above the minimum wage ( $\$ 11.25$ / hour) were compared to see how a one dollar increase in hourly earnings might affect the lifetime payment of income taxes and the breakeven point for the hypothetical citizen. The breakeven point for the citizen is the point where government transfer payments for the citizen are equal to the taxes paid by the citizen. Consequently, the breakeven age is the age when the citizen contributes taxes equivalent to government transfer payments. The analysis was based on the assumptions that work was not interruption due to illness, job loss, or other reasons for the two hypothetical citizens during their working life, and that the two citizens are at the same age. 


\section{RESEARCH RESULTS}

Table 1. Lifetime Taxes Paid by a Citizen Starting at $\$ 10.25$ / hour

and a $2 \%$ Annual Increase in the Canadian Provinces of B.C., Alberta, Sask., Manitoba, Ontario, \& Quebec.

\begin{tabular}{|c|c|c|c|c|c|c|c|}
\hline Age & B.C. & Alberta & Sask. & Manitoba & Ontario & Quebec & Annual Income \\
\hline 1 & $\$ 0.00$ & $\$ 0.00$ & $\$ 0.00$ & $\mathbf{\$ 0 . 0 0}$ & $\$ 0.00$ & $\$ 0.00$ & $\$ 0.00$ \\
\hline 2 & $\$ 0.00$ & $\$ 0.00$ & $\$ 0.00$ & $\$ 0.00$ & $\$ 0.00$ & $\$ 0.00$ & $\$ 0.00$ \\
\hline 3 & $\$ \mathbf{\$ 0 . 0 0}$ & $\$ 0.00$ & $\$ \mathbf{\$ 0 . 0 0}$ & $\$ 0.00$ & $\$ 0.00$ & $\$ 0.00$ & $\$ \mathbf{\$ 0 . 0 0}$ \\
\hline 4 & $\$ \mathbf{\$ 0 . 0 0}$ & $\$ 0.00$ & $\$ 0.00$ & $\$ 0.00$ & $\$ 0.00$ & $\$ 0.00$ & $\$ \mathbf{\$ 0 . 0 0}$ \\
\hline 5 & $\$ 0.00$ & $\$ 0.00$ & $\$ 0.00$ & $\$ 0.00$ & $\$ 0.00$ & $\$ 0.00$ & $\$ 0.00$ \\
\hline 6 & $\$ 0.00$ & $\$ 0.00$ & $\$ 0.00$ & $\$ 0.00$ & $\$ 0.00$ & $\$ 0.00$ & $\$ 0.00$ \\
\hline 7 & $\$ 0.00$ & $\$ 0.00$ & $\$ \mathbf{\$ 0 . 0 0}$ & $\$ 0.00$ & $\$ 0.00$ & $\$ 0.00$ & $\$ 0.00$ \\
\hline 8 & $\$ \mathbf{\$ 0 . 0 0}$ & $\$ 0.00$ & $\$ 0.00$ & $\$ 0.00$ & $\$ 0.00$ & $\$ 0.00$ & $\$ 0.00$ \\
\hline 9 & $\$ 0.00$ & $\$ 0.00$ & $\$ 0.00$ & $\$ 0.00$ & $\$ 0.00$ & $\$ 0.00$ & $\$ 0.00$ \\
\hline 10 & $\$ 0.00$ & $\$ 0.00$ & $\$ 0.00$ & $\$ 0.00$ & $\$ 0.00$ & $\$ 0.00$ & $\$ 0.00$ \\
\hline 11 & $\$ 0.00$ & $\$ 0.00$ & $\$ 0.00$ & $\$ 0.00$ & $\$ 0.00$ & $\$ 0.00$ & $\$ 0.00$ \\
\hline 12 & $\$ 0.00$ & $\$ 0.00$ & $\$ 0.00$ & $\$ 0.00$ & $\$ 0.00$ & $\$ 0.00$ & $\$ 0.00$ \\
\hline 13 & $\$ 0.00$ & $\$ 0.00$ & $\$ 0.00$ & $\$ 0.00$ & $\$ 0.00$ & $\$ 0.00$ & $\$ 0.00$ \\
\hline 14 & $\$ 0.00$ & $\$ 0.00$ & $\$ 0.00$ & $\$ 0.00$ & $\$ 0.00$ & $\$ 0.00$ & $\$ 0.00$ \\
\hline 15 & $\$ 0.00$ & $\$ 0.00$ & $\$ 0.00$ & $\$ 0.00$ & $\$ 0.00$ & $\$ 0.00$ & $\$ 0.00$ \\
\hline 16 & $\$ 0.00$ & $\$ 0.00$ & $\$ 0.00$ & $\$ 0.00$ & $\$ 0.00$ & $\$ 0.00$ & $\$ 0.00$ \\
\hline 17 & $\$ 0.00$ & $\$ 0.00$ & $\$ 0.00$ & $\$ 0.00$ & $\$ 0.00$ & $\$ 0.00$ & $\$ 0.00$ \\
\hline 18 & $\$ 0.00$ & $\$ 0.00$ & $\$ 0.00$ & $\$ 0.00$ & $\$ 0.00$ & $\$ 0.00$ & $\$ \mathbf{\$ 0 . 0 0}$ \\
\hline 19 & $\$ 1,785$ & $\$ 1,978$ & $\$ 2,276$ & $\$ 2,945$ & $\$ 2,176$ & $\$ 2,541$ & $\$ 21,320.00$ \\
\hline 20 & $\$ 1,884$ & $\$ 2,085$ & $\$ 2,387$ & $\$ 3,054$ & $\$ 2,262$ & $\$ 2,662$ & $\$ 21,746.40$ \\
\hline 21 & $\$ 1,985$ & $\$ 2,194$ & $\$ 2,500$ & $\$ 3,167$ & $\$ 2,349$ & $\$ 2,786$ & $\$ 22,181.33$ \\
\hline 22 & $\$ 2,088$ & $\$ 2,304$ & $\$ 2,615$ & $\$ 3,281$ & $\$ 2,438$ & $\$ 2,913$ & $\$ 22,624.95$ \\
\hline 23 & $\$ 2,193$ & $\$ 2,418$ & $\$ 2,733$ & $\$ 3,398$ & $\$ 2,528$ & $\$ 3,042$ & $\$ 23,077.45$ \\
\hline 24 & $\$ 2,301$ & $\$ 2,533$ & $\$ 2,853$ & $\$ 3,517$ & $\$ 2,621$ & $\$ 3,174$ & $\$ 23,539.00$ \\
\hline 25 & $\$ 2,410$ & $\$ 2,651$ & $\$ 2,975$ & $\$ 3,638$ & $\$ 2,715$ & $\$ 3,308$ & $\$ 24,009.78$ \\
\hline 26 & $\$ 2,522$ & $\$ 2,771$ & $\$ 3,100$ & $\$ 3,762$ & $\$ 2,812$ & $\$ 3,445$ & $\$ 24,489.98$ \\
\hline 27 & $\$ 2,636$ & $\$ 2,893$ & $\$ 3,227$ & $\$ 3,889$ & $\$ 2,910$ & $\$ 3,585$ & $\$ 24,979.78$ \\
\hline 28 & $\$ 2,752$ & $\$ 3,018$ & $\$ 3,357$ & $\$ 4,018$ & $\$ 3,010$ & $\$ 3,727$ & $\$ 25,479.37$ \\
\hline 29 & $\$ 2,871$ & $\$ 3,145$ & $\$ 3,490$ & $\$ 4,149$ & $\$ 3,112$ & $\$ 3,872$ & $\$ 25,988.96$ \\
\hline 30 & $\$ 2,991$ & $\$ 3,275$ & $\$ 3,625$ & $\$ 4,283$ & $\$ 3,216$ & $\$ 4,021$ & $\$ 26,508.74$ \\
\hline 31 & $\$ 3,115$ & $\$ 3,408$ & $\$ 3,763$ & $\$ 4,420$ & $\$ 3,323$ & $\$ 4,172$ & $\$ 27,038.92$ \\
\hline 32 & $\$ 3,241$ & $\$ 3,543$ & $\$ 3,903$ & $\$ 4,559$ & $\$ 3,431$ & $\$ 4,326$ & $\$ 27,579.69$ \\
\hline 33 & $\$ 3,369$ & $\$ 3,681$ & $\$ 4,047$ & $\$ 4,702$ & $\$ 3,542$ & $\$ 4,484$ & $\$ 28,131.29$ \\
\hline 34 & $\$ 3,500$ & $\$ 3,822$ & $\$ 4,193$ & $\$ 4,847$ & $\$ 3,655$ & $\$ 4,644$ & $\$ 28,693.91$ \\
\hline 35 & $\$ 3,633$ & $\$ 3,965$ & $\$ 4,342$ & $\$ 4,995$ & $\$ 3,770$ & $\$ 4,808$ & $\$ 29,267.79$ \\
\hline 36 & $\$ 3,770$ & $\$ 4,112$ & $\$ 4,495$ & $\$ 5,146$ & $\$ 3,887$ & $\$ 4,975$ & $\$ 29,853.15$ \\
\hline 37 & $\$ 3,908$ & $\$ 4,261$ & $\$ 4,650$ & $\$ 5,300$ & $\$ 4,007$ & $\$ 5,145$ & $\$ 30,450.21$ \\
\hline 38 & $\$ 4,032$ & $\$ 4,413$ & $\$ 4,808$ & $\$ 5,458$ & $\$ 4,129$ & $\$ 5,319$ & $\$ 31,059.21$ \\
\hline 39 & $\$ 4,157$ & $\$ 4,568$ & $\$ 4,970$ & $\$ 5,631$ & $\$ 4,253$ & $\$ 5,496$ & $\$ 31,680.40$ \\
\hline 40 & $\$ 4,284$ & $\$ 4,727$ & $\$ 5,134$ & $\$ 5,807$ & $\$ 4,381$ & $\$ 5,677$ & $\$ 32,314.01$ \\
\hline 41 & $\$ 4,414$ & $\$ 4,888$ & $\$ 5,302$ & $\$ 5,986$ & $\$ 4,510$ & $\$ 5,861$ & $\$ 32,960.29$ \\
\hline 42 & $\$ 4,546$ & $\$ 5,053$ & $\$ 5,474$ & $\$ 6,169$ & $\$ 4,642$ & $\$ 6,049$ & $\$ 33,619.49$ \\
\hline 43 & $\$ 4,681$ & $\$ 5,221$ & $\$ 5,648$ & $\$ 6,355$ & $\$ 4,777$ & $\$ 6,241$ & $\$ 34,291.88$ \\
\hline 44 & $\$ 4,818$ & $\$ 5,393$ & $\$ 5,827$ & $\$ 6,546$ & $\$ 4,914$ & $\$ 6,437$ & $\$ 34,977.72$ \\
\hline 45 & $\$ 4,959$ & $\$ 5,568$ & $\$ 6,009$ & $\$ 6,740$ & $\$ 5,055$ & $\$ 6,636$ & $\$ 35,677.27$ \\
\hline 46 & $\$ 5,102$ & $\$ 5,746$ & $\$ 6,194$ & $\$ 6,938$ & $\$ 5,198$ & $\$ 6,840$ & $\$ 36,390.82$ \\
\hline 47 & $\$ 5,251$ & $\$ 5,928$ & $\$ 6,384$ & $\$ 7,140$ & $\$ 5,344$ & $\$ 7,047$ & $\$ 37,118.64$ \\
\hline 48 & $\$ 5,419$ & $\$ 6,114$ & $\$ 6,577$ & $\$ 7,346$ & $\$ 5,493$ & $\$ 7,259$ & $\$ 37,861.01$ \\
\hline 49 & $\$ 5,591$ & $\$ 6,303$ & $\$ 6,774$ & $\$ 7,556$ & $\$ 5,644$ & $\$ 7,475$ & $\$ 38,618.23$ \\
\hline 50 & $\$ 5,766$ & $\$ 6,496$ & $\$ 6,974$ & $\$ 7,770$ & $\$ 5,814$ & $\$ 7,695$ & $\$ 39,390.59$ \\
\hline 51 & $\$ 5,945$ & $\$ 6,693$ & $\$ 7,179$ & $\$ 7,989$ & $\$ 6,005$ & $\$ 7,923$ & $\$ 40,178.41$ \\
\hline 52 & $\$ 6,128$ & $\$ 6,894$ & $\$ 7,388$ & $\$ 8,212$ & $\$ 6,199$ & $\$ 8,184$ & $\$ 40,981.97$ \\
\hline 53 & $\$ 6,314$ & $\$ 7,099$ & $\$ 7,601$ & $\$ 8,439$ & $\$ 6,397$ & $\$ 8,451$ & $\$ 41,801.61$ \\
\hline 54 & $\$ 6,503$ & $\$ 7,308$ & $\$ 7,830$ & $\$ 8,671$ & $\$ 6,599$ & $\$ 8,723$ & $\$ 42,637.65$ \\
\hline 55 & $\$ 6,752$ & $\$ 7,575$ & $\$ 8,123$ & $\$ 8,963$ & $\$ 6,859$ & $\$ 9,046$ & $\$ 43,490.40$ \\
\hline 56 & $\$ 7,010$ & $\$ 7,854$ & $\$ 8,428$ & $\$ 9,265$ & $\$ 7,130$ & $\$ 9,380$ & $\$ 44,360.21$ \\
\hline
\end{tabular}


$\underline{\text { (Table } 1 \text { continued) }}$

\begin{tabular}{|c|c|c|c|c|c|c|c|}
\hline Age & B.C. & Alberta & Sask. & Manitoba & Ontario & Quebec & Annual Income \\
\hline 57 & $\$ 7,274$ & $\$ 8,138$ & $\$ 8,738$ & $\$ 9,573$ & $\$ 7,407$ & $\$ 9,720$ & $\$ 45,247.41$ \\
\hline 58 & $\$ 7,542$ & $\$ 8,427$ & $\$ 9,055$ & $\$ 9,888$ & $\$ 7,688$ & $\$ 10,067$ & $\$ 46,152.36$ \\
\hline 59 & $\$ 7,817$ & $\$ 8,723$ & $\$ 9,378$ & $\$ 10,208$ & $\$ 7,976$ & $\$ 10,422$ & $\$ 47,075.41$ \\
\hline 60 & $\$ 8,096$ & $\$ 9,024$ & $\$ 9,708$ & $\$ 10,535$ & $\$ 8,269$ & $\$ 10,783$ & $\$ 48,016.91$ \\
\hline 61 & $\$ 8,381$ & $\$ 9,331$ & $\$ 10,044$ & $\$ 10,869$ & $\$ 8,568$ & $\$ 11,151$ & $\$ 48,977.25$ \\
\hline 62 & $\$ 8,672$ & $\$ 9,645$ & $\$ 10,387$ & $\$ 11,210$ & $\$ 8,873$ & $\$ 11,527$ & $\$ 49,956.80$ \\
\hline 63 & $\$ 8,969$ & $\$ 9,964$ & $\$ 10,736$ & $\$ 11,557$ & $\$ 9,185$ & $\$ 11,910$ & $\$ 50,955.93$ \\
\hline 64 & $\$ 9,272$ & $\$ 10,291$ & $\$ 11,093$ & $\$ 11,911$ & $\$ 9,502$ & $\$ 12,302$ & $\$ 51,975.05$ \\
\hline 65 & $\$ 9,580$ & $\$ 10,623$ & $\$ 11,457$ & $\$ 12,272$ & $\$ 9,826$ & $\$ 12,700$ & $\$ 53,014.55$ \\
\hline 66 & $\$ 9,895$ & $\$ 10,962$ & $\$ 11,828$ & $\$ 12,641$ & $\$ 10,156$ & $\$ 13,107$ & $\$ 54,074.84$ \\
\hline 67 & $\$ 10,217$ & $\$ 11,309$ & $\$ 12,207$ & $\$ 13,017$ & $\$ 10,493$ & $\$ 13,522$ & $\$ 55,156.34$ \\
\hline 68 & $\$ 0.00$ & $\$ 0.00$ & $\$ 0.00$ & $\$ 0.00$ & $\$ 0.00$ & $\$ 0.00$ & $\$ 0.00$ \\
\hline 69 & $\$ 0.00$ & $\$ 0.00$ & $\$ 0.00$ & $\$ 0.00$ & $\$ 0.00$ & $\$ 0.00$ & $\$ 0.00$ \\
\hline 70 & $\$ 0.00$ & $\$ 0.00$ & $\$ 0.00$ & $\$ 0.00$ & $\$ 0.00$ & $\$ 0.00$ & $\$ 0.00$ \\
\hline 71 & $\$ 0.00$ & $\$ 0.00$ & $\$ 0.00$ & $\$ 0.00$ & $\$ 0.00$ & $\$ 0.00$ & $\$ 0.00$ \\
\hline 72 & $\$ 0.00$ & $\$ 0.00$ & $\$ 0.00$ & $\$ 0.00$ & $\$ 0.00$ & $\$ 0.00$ & $\$ 0.00$ \\
\hline 73 & $\$ 0.00$ & $\$ 0.00$ & $\$ 0.00$ & $\$ \mathbf{\$ 0 . 0 0}$ & $\$ 0.00$ & $\$ 0.00$ & $\$ 0.00$ \\
\hline 74 & $\$ 0.00$ & $\$ 0.00$ & $\$ 0.00$ & $\$ 0.00$ & $\$ \mathbf{\$ 0 . 0 0}$ & $\$ 0.00$ & $\$ 0.00$ \\
\hline 75 & $\$ 0.00$ & $\$ 0.00$ & $\$ 0.00$ & $\$ 0.00$ & $\$ 0.00$ & $\$ 0.00$ & $\$ 0.00$ \\
\hline 76 & $\$ 0.00$ & $\$ 0.00$ & $\$ 0.00$ & $\$ 0.00$ & $\$ 0.00$ & $\$ 0.00$ & $\$ 0.00$ \\
\hline 77 & $\$ 0.00$ & $\$ 0.00$ & $\$ 0.00$ & $\$ 0.00$ & $\$ 0.00$ & $\$ 0.00$ & $\$ 0.00$ \\
\hline 78 & $\$ 0.00$ & $\$ 0.00$ & $\$ 0.00$ & $\$ 0.00$ & $\$ 0.00$ & $\$ 0.00$ & $\$ 0.00$ \\
\hline 79 & $\$ 0.00$ & $\$ 0.00$ & $\$ 0.00$ & $\$ 0.00$ & $\$ 0.00$ & $\$ 0.00$ & $\$ 0.00$ \\
\hline 80 & $\$ 0.00$ & $\$ 0.00$ & $\$ 0.00$ & $\$ 0.00$ & $\$ 0.00$ & $\$ 0.00$ & $\$ 0.00$ \\
\hline Total & $\$ 250,341$ & $\$ 278,335$ & $\$ 301,786$ & $\$ 337,732$ & $\$ 259,050$ & $\$ 334,580$ & $\$ 1,746,973$ \\
\hline
\end{tabular}

Table 2. Lifetime Taxes Paid by a Citizen Starting at $\$ 10.25 /$ hour

and a $2 \%$ Annual Increase in the Canadian Provinces of N.B, N.S., P.E.I., Nfld., N.W.T., Yukon, \& Nunavut.

\begin{tabular}{|c|c|c|c|c|c|c|c|c|}
\hline Age & N.B. & N.S. & P.E.I. & Nfld. & N.W.T. & Yukon & Nunavut & Annual Income \\
\hline 1 & $\$ \mathbf{\$ 0 . 0 0}$ & $\$ \mathbf{\$ 0 . 0 0}$ & $\$ \mathbf{\$ 0 . 0 0}$ & $\$ \mathbf{\$ 0 . 0 0}$ & $\$ 0.00$ & $\$ 0.00$ & $\$ 0.00$ & $\mathbf{\$ 0 . 0 0}$ \\
\hline 2 & $\$ 0.00$ & $\$ 0.00$ & $\$ 0.00$ & $\$ 0.00$ & $\$ 0.00$ & $\$ \mathbf{\$ 0 . 0 0}$ & $\$ 0.00$ & $\$ \mathbf{\$ 0 . 0 0}$ \\
\hline 3 & $\$ 0.00$ & $\$ 0.00$ & $\$ 0.00$ & $\$ 0.00$ & $\$ 0.00$ & $\$ 0.00$ & $\$ 0.00$ & $\$ \mathbf{\$ 0 . 0 0}$ \\
\hline 4 & $\$ 0.00$ & $\$ 0.00$ & $\$ 0.00$ & $\$ 0.00$ & $\$ 0.00$ & $\$ 0.00$ & $\$ 0.00$ & $\$ 0.00$ \\
\hline 5 & $\$ 0.00$ & $\$ 0.00$ & $\$ 0.00$ & $\$ 0.00$ & $\$ 0.00$ & $\$ 0.00$ & $\$ 0.00$ & $\$ 0.00$ \\
\hline 6 & $\$ 0.00$ & $\$ 0.00$ & $\$ 0.00$ & $\$ 0.00$ & $\$ 0.00$ & $\$ \mathbf{\$ 0 . 0 0}$ & $\$ \mathbf{\$ 0 . 0 0}$ & $\$ \mathbf{\$ 0 . 0 0}$ \\
\hline 7 & $\$ 0.00$ & $\$ 0.00$ & $\$ 0.00$ & $\$ 0.00$ & $\$ 0.00$ & $\$ 0.00$ & $\$ 0.00$ & $\$ 0.00$ \\
\hline 8 & $\$ 0.00$ & $\$ 0.00$ & $\$ 0.00$ & $\$ 0.00$ & $\$ 0.00$ & $\$ 0.00$ & $\$ \mathbf{\$ 0 . 0 0}$ & $\$ 0.00$ \\
\hline 9 & $\$ 0.00$ & $\$ 0.00$ & $\$ 0.00$ & $\$ 0.00$ & $\$ 0.00$ & $\$ 0.00$ & $\$ 0.00$ & $\$ 0.00$ \\
\hline 10 & $\$ 0.00$ & $\$ 0.00$ & $\$ 0.00$ & $\$ 0.00$ & $\$ 0.00$ & $\$ \mathbf{\$ 0 . 0 0}$ & $\$ \mathbf{\$ 0 . 0 0}$ & $\$ 0.00$ \\
\hline 11 & $\$ \mathbf{\$ 0 . 0 0}$ & $\$ 0.00$ & $\$ 0.00$ & $\$ 0.00$ & $\$ 0.00$ & $\$ \mathbf{\$ 0 . 0 0}$ & $\$ 0.00$ & $\$ 0.00$ \\
\hline 12 & $\$ 0.00$ & $\$ 0.00$ & $\$ 0.00$ & $\$ 0.00$ & $\$ 0.00$ & $\$ 0.00$ & $\$ 0.00$ & $\$ 0.00$ \\
\hline 13 & $\$ 0.00$ & $\$ 0.00$ & $\$ 0.00$ & $\$ 0.00$ & $\$ 0.00$ & $\$ 0.00$ & $\$ 0.00$ & $\$ 0.00$ \\
\hline 14 & $\$ 0.00$ & $\$ 0.00$ & $\$ 0.00$ & $\$ 0.00$ & $\$ 0.00$ & $\$ 0.00$ & $\$ 0.00$ & $\$ 0.00$ \\
\hline 15 & $\$ 0.00$ & $\$ 0.00$ & $\$ 0.00$ & $\$ 0.00$ & $\$ 0.00$ & $\$ 0.00$ & $\$ 0.00$ & $\$ 0.00$ \\
\hline 16 & $\$ 0.00$ & $\$ 0.00$ & $\$ 0.00$ & $\$ 0.00$ & $\$ 0.00$ & $\$ 0.00$ & $\$ 0.00$ & $\$ 0.00$ \\
\hline 17 & $\$ 0.00$ & $\$ 0.00$ & $\$ 0.00$ & $\$ 0.00$ & $\$ 0.00$ & $\$ 0.00$ & $\$ 0.00$ & $\$ 0.00$ \\
\hline 18 & $\$ 0.00$ & $\$ \mathbf{\$ 0 . 0 0}$ & $\$ 0.00$ & $\$ \mathbf{\$ 0 . 0 0}$ & $\$ 0.00$ & $\$ 0.00$ & $\$ \mathbf{\$ 0 . 0 0}$ & $\$ 0.00$ \\
\hline 19 & $\$ 2,296$ & $\$ 2,703$ & $\$ 2,908$ & $\$ 2,508$ & $\$ 2,049$ & $\$ 2,314$ & $\$ 1,939$ & $\$ 21,320.00$ \\
\hline 20 & $\$ 2,411$ & $\$ 2,804$ & $\$ 3,014$ & $\$ 2,604$ & $\$ 2,138$ & $\$ 2,407$ & $\$ 2,020$ & $\$ 21,746.40$ \\
\hline 21 & $\$ 2,529$ & $\$ 2,908$ & $\$ 3,122$ & $\$ 2,703$ & $\$ 2,229$ & $\$ 2,503$ & $\$ 2,102$ & $\$ 22,181.33$ \\
\hline 22 & $\$ 2,649$ & $\$ 3,013$ & $\$ 3,232$ & $\$ 2,804$ & $\$ 2,321$ & $\$ 2,601$ & $\$ 2,187$ & $\$ 22,624.95$ \\
\hline 23 & $\$ 2,772$ & $\$ 3,121$ & $\$ 3,344$ & $\$ 2,907$ & $\$ 2,416$ & $\$ 2,701$ & $\$ 2,273$ & $\$ 23,077.45$ \\
\hline 24 & $\$ 2,897$ & $\$ 3,231$ & $\$ 3,459$ & $\$ 3,012$ & $\$ 2,513$ & $\$ 2,803$ & $\$ 2,360$ & $\$ 23,539.00$ \\
\hline 25 & $\$ 3,024$ & $\$ 3,343$ & $\$ 3,575$ & $\$ 3,118$ & $\$ 2,611$ & $\$ 2,906$ & $\$ 2,450$ & $\$ 24,009.78$ \\
\hline 26 & $\$ 3,155$ & $\$ 3,457$ & $\$ 3,694$ & $\$ 3,227$ & $\$ 2,711$ & $\$ 3,012$ & $\$ 2,541$ & $\$ 24,489.98$ \\
\hline 27 & $\$ 3,287$ & $\$ 3,573$ & $\$ 3,816$ & $\$ 3,338$ & $\$ 2,814$ & $\$ 3,120$ & $\$ 2,634$ & $\$ 24,979.78$ \\
\hline 28 & $\$ 3,423$ & $\$ 3,692$ & $\$ 3,940$ & $\$ 3,452$ & $\$ 2,918$ & $\$ 3,230$ & $\$ 2,729$ & $\$ 25,479.37$ \\
\hline 29 & $\$ 3,561$ & $\$ 3,814$ & $\$ 4,066$ & $\$ 3,567$ & $\$ 3,024$ & $\$ 3,342$ & $\$ 2,826$ & $\$ 25,988.96$ \\
\hline
\end{tabular}


(Table 2 continued)

\begin{tabular}{|c|c|c|c|c|c|c|c|c|}
\hline Age & N.B. & N.S. & P.E.I. & Nfld. & N.W.T. & Yukon & Nunavut & Annual Income \\
\hline 30 & $\$ 3,702$ & $\$ 3,937$ & $\$ 4,195$ & $\$ 3,685$ & $\$ 3,133$ & $\$ 3,457$ & $\$ 2,925$ & $\$ 26,508.74$ \\
\hline 31 & $\$ 3,845$ & $\$ 4,063$ & $\$ 4,326$ & $\$ 3,806$ & $\$ 3,244$ & $\$ 3,574$ & $\$ 3,025$ & $\$ 27,038.92$ \\
\hline 32 & $\$ 3,992$ & $\$ 4,192$ & $\$ 4,461$ & $\$ 3,929$ & $\$ 3,357$ & $\$ 3,693$ & $\$ 3,128$ & $\$ 27,579.69$ \\
\hline 33 & $\$ 4,141$ & $\$ 4,323$ & $\$ 4,598$ & $\$ 4,054$ & $\$ 3,472$ & $\$ 3,815$ & $\$ 3,233$ & $\$ 28,131.29$ \\
\hline 34 & $\$ 4,294$ & $\$ 4,457$ & $\$ 4,737$ & $\$ 4,181$ & $\$ 3,590$ & $\$ 3,939$ & $\$ 3,340$ & $\$ 28,693.91$ \\
\hline 35 & $\$ 4,449$ & $\$ 4,594$ & $\$ 4,879$ & $\$ 4,312$ & $\$ 3,710$ & $\$ 4,065$ & $\$ 3,449$ & $\$ 29,267.79$ \\
\hline 36 & $\$ 4,608$ & $\$ 4,749$ & $\$ 5,025$ & $\$ 4,445$ & $\$ 3,832$ & $\$ 4,194$ & $\$ 3,560$ & $\$ 29,853.15$ \\
\hline 37 & $\$ 4,770$ & $\$ 4,928$ & $\$ 5,173$ & $\$ 4,580$ & $\$ 3,957$ & $\$ 4,326$ & $\$ 3,674$ & $\$ 30,450.21$ \\
\hline 38 & $\$ 4,935$ & $\$ 5,110$ & $\$ 5,324$ & $\$ 4,719$ & $\$ 4,084$ & $\$ 4,460$ & $\$ 3,789$ & $\$ 31,059.21$ \\
\hline 39 & $\$ 5,103$ & $\$ 5,296$ & $\$ 5,478$ & $\$ 4,860$ & $\$ 4,214$ & $\$ 4,597$ & $\$ 3,907$ & $\$ 31,680.40$ \\
\hline 40 & $\$ 5,275$ & $\$ 5,486$ & $\$ 5,648$ & $\$ 5,003$ & $\$ 4,347$ & $\$ 4,737$ & $\$ 4,028$ & $\$ 32,314.01$ \\
\hline 41 & $\$ 5,450$ & $\$ 5,680$ & $\$ 5,834$ & $\$ 5,153$ & $\$ 4,482$ & $\$ 4,879$ & $\$ 4,150$ & $\$ 32,960.29$ \\
\hline 42 & $\$ 5,629$ & $\$ 5,877$ & $\$ 6,024$ & $\$ 5,334$ & $\$ 4,619$ & $\$ 5,024$ & $\$ 4,276$ & $\$ 33,619.49$ \\
\hline 43 & $\$ 5,803$ & $\$ 6,078$ & $\$ 6,217$ & $\$ 5,519$ & $\$ 4,760$ & $\$ 5,172$ & $\$ 4,403$ & $\$ 34,291.88$ \\
\hline 44 & $\$ 5,968$ & $\$ 6,284$ & $\$ 6,415$ & $\$ 5,708$ & $\$ 4,903$ & $\$ 5,324$ & $\$ 4,534$ & $\$ 34,977.72$ \\
\hline 45 & $\$ 6,137$ & $\$ 6,493$ & $\$ 6,617$ & $\$ 5,900$ & $\$ 5,049$ & $\$ 5,478$ & $\$ 4,667$ & $\$ 35,677.27$ \\
\hline 46 & $\$ 6,309$ & $\$ 6,707$ & $\$ 6,822$ & $\$ 6,096$ & $\$ 5,198$ & $\$ 5,635$ & $\$ 4,802$ & $\$ 36,390.82$ \\
\hline 47 & $\$ 6,484$ & $\$ 6,925$ & $\$ 7,032$ & $\$ 6,297$ & $\$ 5,351$ & $\$ 5,795$ & $\$ 4,940$ & $\$ 37,118.64$ \\
\hline 48 & $\$ 6,664$ & $\$ 7,148$ & $\$ 7,246$ & $\$ 6,501$ & $\$ 5,506$ & $\$ 5,959$ & $\$ 5,082$ & $\$ 37,861.01$ \\
\hline 49 & $\$ 6,859$ & $\$ 7,374$ & $\$ 7,464$ & $\$ 6,709$ & $\$ 5,664$ & $\$ 6,126$ & $\$ 5,225$ & $\$ 38,618.23$ \\
\hline 50 & $\$ 7,068$ & $\$ 7,605$ & $\$ 7,686$ & $\$ 6,921$ & $\$ 5,845$ & $\$ 6,296$ & $\$ 5,372$ & $\$ 39,390.59$ \\
\hline 51 & $\$ 7,281$ & $\$ 7,841$ & $\$ 7,913$ & $\$ 7,138$ & $\$ 6,031$ & $\$ 6,470$ & $\$ 5,522$ & $\$ 40,178.41$ \\
\hline 52 & $\$ 7,499$ & $\$ 8,082$ & $\$ 8,144$ & $\$ 7,359$ & $\$ 6,220$ & $\$ 6,647$ & $\$ 5,682$ & $\$ 40,981.97$ \\
\hline 53 & $\$ 7,721$ & $\$ 8,328$ & $\$ 8,380$ & $\$ 7,584$ & $\$ 6,414$ & $\$ 6,828$ & $\$ 5,863$ & $\$ 41,801.61$ \\
\hline 54 & $\$ 7,948$ & $\$ 8,578$ & $\$ 8,621$ & $\$ 7,814$ & $\$ 6,611$ & $\$ 7,012$ & $\$ 6,047$ & $\$ 42,637.65$ \\
\hline 55 & $\$ 8,234$ & $\$ 8,888$ & $\$ 8,922$ & $\$ 8,104$ & $\$ 6,867$ & $\$ 7,275$ & $\$ 6,289$ & $\$ 43,490.40$ \\
\hline 56 & $\$ 8,530$ & $\$ 9,210$ & $\$ 9,233$ & $\$ 8,404$ & $\$ 7,133$ & $\$ 7,551$ & $\$ 6,541$ & $\$ 44,360.21$ \\
\hline 57 & $\$ 8,833$ & $\$ 9,537$ & $\$ 9,551$ & $\$ 8,710$ & $\$ 7,405$ & $\$ 7,832$ & $\$ 6,798$ & $\$ 45,247.41$ \\
\hline 58 & $\$ 9,142$ & $\$ 9,872$ & $\$ 9,875$ & $\$ 9,022$ & $\$ 7,682$ & $\$ 8,119$ & $\$ 7,061$ & $\$ 46,152.36$ \\
\hline 59 & $\$ 9,456$ & $\$ 10,213$ & $\$ 10,205$ & $\$ 9,341$ & $\$ 7,964$ & $\$ 8,411$ & $\$ 7,329$ & $\$ 47,075.41$ \\
\hline 60 & $\$ 9,777$ & $\$ 10,561$ & $\$ 10,542$ & $\$ 9,665$ & $\$ 8,252$ & $\$ 8,709$ & $\$ 7,601$ & $\$ 48,016.91$ \\
\hline 61 & $\$ 10,105$ & $\$ 10,916$ & $\$ 10,886$ & $\$ 9,997$ & $\$ 8,546$ & $\$ 9,013$ & $\$ 7,880$ & $\$ 48,977.25$ \\
\hline 62 & $\$ 10,439$ & $\$ 11,277$ & $\$ 11,236$ & $\$ 10,334$ & $\$ 8,846$ & $\$ 9,324$ & $\$ 8,164$ & $\$ 49,956.80$ \\
\hline 63 & $\$ 10,779$ & $\$ 11,646$ & $\$ 11,594$ & $\$ 10,679$ & $\$ 9,151$ & $\$ 9,640$ & $\$ 8,454$ & $\$ 50,955.93$ \\
\hline 64 & $\$ 11,127$ & $\$ 12,023$ & $\$ 11,959$ & $\$ 11,031$ & $\$ 9,463$ & $\$ 9,963$ & $\$ 8,750$ & $\$ 51,975.05$ \\
\hline 65 & $\$ 11,481$ & $\$ 12,407$ & $\$ 12,331$ & $\$ 11,389$ & $\$ 9,781$ & $\$ 10,292$ & $\$ 9,051$ & $\$ 53,014.55$ \\
\hline 66 & $\$ 11,843$ & $\$ 12,799$ & $\$ 12,711$ & $\$ 11,755$ & $\$ 10,106$ & $\$ 10,628$ & $\$ 9,358$ & $\$ 54,074.84$ \\
\hline 67 & $\$ 12,212$ & $\$ 13,199$ & $\$ 13,098$ & $\$ 12,128$ & $\$ 10,437$ & $\$ 10,971$ & $\$ 9,672$ & $\$ 55,156.34$ \\
\hline 68 & $\$ 0.00$ & $\$ 0.00$ & $\$ 0.00$ & $\$ 0.00$ & $\$ 0.00$ & $\$ 0.00$ & $\$ 0.00$ & $\$ 0.00$ \\
\hline 69 & $\$ 0.00$ & $\$ 0.00$ & $\$ 0.00$ & $\$ 0.00$ & $\$ 0.00$ & $\$ 0.00$ & $\$ 0.00$ & $\$ 0.00$ \\
\hline 70 & $\$ 0.00$ & $\$ 0.00$ & $\$ 0.00$ & $\$ 0.00$ & $\$ 0.00$ & $\$ 0.00$ & $\$ 0.00$ & $\$ 0.00$ \\
\hline 71 & $\$ 0.00$ & $\$ 0.00$ & $\$ 0.00$ & $\$ 0.00$ & $\$ 0.00$ & $\$ 0.00$ & $\$ 0.00$ & $\$ 0.00$ \\
\hline 72 & $\$ \mathbf{\$ 0 . 0 0}$ & $\$ 0.00$ & $\$ 0.00$ & $\$ 0.00$ & $\$ 0.00$ & $\$ 0.00$ & $\$ 0.00$ & $\$ 0.00$ \\
\hline 73 & $\$ 0.00$ & $\$ 0.00$ & $\$ 0.00$ & $\$ 0.00$ & $\$ 0.00$ & $\$ 0.00$ & $\$ 0.00$ & $\$ 0.00$ \\
\hline 74 & $\$ 0.00$ & $\$ 0.00$ & $\$ 0.00$ & $\$ 0.00$ & $\$ 0.00$ & $\$ 0.00$ & $\$ 0.00$ & $\$ 0.00$ \\
\hline 75 & $\$ 0.00$ & $\$ 0.00$ & $\$ 0.00$ & $\$ 0.00$ & $\$ 0.00$ & $\$ \mathbf{\$ 0 . 0 0}$ & $\$ 0.00$ & $\$ 0.00$ \\
\hline 76 & $\$ 0.00$ & $\$ 0.00$ & $\$ 0.00$ & $\$ 0.00$ & $\$ 0.00$ & $\$ 0.00$ & $\$ 0.00$ & $\$ 0.00$ \\
\hline 77 & $\$ \mathbf{\$ 0 . 0 0}$ & $\$ 0.00$ & $\$ 0.00$ & $\$ 0.00$ & $\$ 0.00$ & $\$ 0.00$ & $\$ 0.00$ & $\$ 0.00$ \\
\hline 78 & $\$ 0.00$ & $\$ 0.00$ & $\$ 0.00$ & $\$ 0.00$ & $\$ 0.00$ & $\$ 0.00$ & $\$ 0.00$ & $\$ 0.00$ \\
\hline 79 & $\$ 0.00$ & $\$ 0.00$ & $\$ 0.00$ & $\$ 0.00$ & $\$ 0.00$ & $\$ \mathbf{\$ 0 . 0 0}$ & $\$ 0.00$ & $\$ 0.00$ \\
\hline 80 & $\$ \mathbf{\$ 0 . 0 0}$ & $\$ \mathbf{\$ 0 . 0 0}$ & $\$ \mathbf{\$ 0 . 0 0}$ & $\$ \mathbf{\$ 0 . 0 0}$ & $\$ 0.00$ & $\$ \mathbf{\$ 0 . 0 0}$ & $\$ 0.00$ & $\$ \mathbf{\$ 0 . 0 0}$ \\
\hline Total & $\$ 305,896$ & $\$ 328,342$ & $\$ 334,572$ & $\$ 301,406$ & $\$ 256,970$ & $\$ 276,169$ & $\$ 237,632$ & $\$ 1,746,973$ \\
\hline
\end{tabular}

Note. Tax calculations are based on average tax rates of 2012 using Ernst \& Young personal income tax calculator. 
Table 3. Lifetime Taxes Paid by a Citizen Starting at $\$ 11.25$ / hour

and a $2 \%$ Annual Increase in the Canadian Provinces of B.C., Alberta, Sask., Manitoba, Ontario, \& Quebec.

\begin{tabular}{|c|c|c|c|c|c|c|c|}
\hline Age & B.C. & Alberta & Sask. & Manitoba & Ontario & Quebec & Annual Income \\
\hline 1 & $\$ 0.00$ & 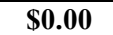 & $\$ 0.00$ & $\$ 0.00$ & $\$ \$ \mathbf{0 . 0 0}$ & $\$ \mathbf{\$ 0 . 0 0}$ & $\mathbf{\$ 0 . 0 0}$ \\
\hline 2 & $\$ 0.00$ & $\$ 0.00$ & $\$ 0.00$ & $\$ 0.00$ & $\$ 0.00$ & $\$ 0.00$ & $\$ 0.00$ \\
\hline 3 & $\$ 0.00$ & $\$ 0.00$ & $\$ 0.00$ & $\$ 0.00$ & $\$ 0.00$ & $\$ 0.00$ & $\$ 0.00$ \\
\hline 4 & $\$ 0.00$ & $\$ 0.00$ & $\$ 0.00$ & $\$ 0.00$ & $\$ 0.00$ & $\$ 0.00$ & $\$ 0.00$ \\
\hline 5 & $\$ 0.00$ & $\$ 0.00$ & $\$ 0.00$ & $\$ 0.00$ & $\$ 0.00$ & $\$ 0.00$ & $\$ 0.00$ \\
\hline 6 & $\$ 0.00$ & $\$ 0.00$ & $\$ 0.00$ & $\$ 0.00$ & $\$ 0.00$ & $\$ 0.00$ & $\$ 0.00$ \\
\hline 7 & $\$ 0.00$ & $\$ 0.00$ & $\$ 0.00$ & $\$ 0.00$ & $\$ 0.00$ & $\$ 0.00$ & $\$ 0.00$ \\
\hline 8 & $\$ 0.00$ & $\$ 0.00$ & $\$ 0.00$ & $\$ 0.00$ & $\$ 0.00$ & $\$ 0.00$ & $\$ 0.00$ \\
\hline 9 & $\$ 0.00$ & $\$ 0.00$ & $\$ 0.00$ & $\$ 0.00$ & $\$ 0.00$ & $\$ 0.00$ & $\$ 0.00$ \\
\hline 10 & $\$ 0.00$ & $\$ 0.00$ & $\$ 0.00$ & $\$ 0.00$ & $\$ 0.00$ & $\$ 0.00$ & $\$ 0.00$ \\
\hline 11 & $\$ 0.00$ & $\$ 0.00$ & $\$ 0.00$ & $\$ 0.00$ & $\$ 0.00$ & $\$ 0.00$ & $\$ 0.00$ \\
\hline 12 & $\$ 0.00$ & $\$ 0.00$ & $\$ 0.00$ & $\$ 0.00$ & $\$ 0.00$ & $\$ 0.00$ & $\$ 0.00$ \\
\hline 13 & $\$ 0.00$ & $\$ 0.00$ & $\$ 0.00$ & $\$ 0.00$ & $\$ 0.00$ & $\$ 0.00$ & $\$ 0.00$ \\
\hline 14 & $\$ 0.00$ & $\$ 0.00$ & $\$ 0.00$ & $\$ 0.00$ & $\$ 0.00$ & $\$ 0.00$ & $\$ 0.00$ \\
\hline 15 & $\$ 0.00$ & $\$ 0.00$ & $\$ 0.00$ & $\$ 0.00$ & $\$ 0.00$ & $\$ 0.00$ & $\$ 0.00$ \\
\hline 16 & $\$ 0.00$ & $\$ 0.00$ & $\$ 0.00$ & $\$ 0.00$ & $\$ 0.00$ & $\$ 0.00$ & $\$ 0.00$ \\
\hline 17 & $\$ 0.00$ & $\$ 0.00$ & $\$ 0.00$ & $\$ 0.00$ & $\$ 0.00$ & $\$ 0.00$ & $\$ 0.00$ \\
\hline 18 & $\$ 0.00$ & $\$ 0.00$ & $\$ \mathbf{\$ 0 . 0 0}$ & $\$ 0.00$ & $\$ 0.00$ & $\$ 0.00$ & $\$ \mathbf{\$ 0 . 0 0}$ \\
\hline 19 & $\$ 2,269$ & $\$ 2,498$ & $\$ 2,817$ & $\$ 3,481$ & $\$ 2,593$ & $\$ 3,134$ & $\$ 23,400.00$ \\
\hline 20 & $\$ 2,377$ & $\$ 2,615$ & $\$ 2,939$ & $\$ 3,602$ & $\$ 2,687$ & $\$ 3,268$ & $\$ 23,868.00$ \\
\hline 21 & $\$ 2,488$ & $\$ 2,735$ & $\$ 3,063$ & $\$ 3,725$ & $\$ 2,783$ & $\$ 3,404$ & $\$ 24,345.36$ \\
\hline 22 & $\$ 2,602$ & $\$ 2,856$ & $\$ 3,189$ & $\$ 3,851$ & $\$ 2,880$ & $\$ 3,543$ & $\$ 24,832.27$ \\
\hline 23 & $\$ 2,717$ & $\$ 2,980$ & $\$ 3,318$ & $\$ 3,979$ & $\$ 2,980$ & $\$ 3,684$ & $\$ 25,328.91$ \\
\hline 24 & $\$ 2,835$ & $\$ 3,107$ & $\$ 3,450$ & $\$ 4,109$ & $\$ 3,081$ & $\$ 3,829$ & $\$ 25,835.49$ \\
\hline 25 & $\$ 2,955$ & $\$ 3,236$ & $\$ 3,584$ & $\$ 4,243$ & $\$ 3,185$ & $\$ 3,976$ & $\$ 26,352.20$ \\
\hline 26 & $\$ 3,078$ & $\$ 3,368$ & $\$ 3,721$ & $\$ 4,379$ & $\$ 3,291$ & $\$ 4,127$ & $\$ 26,879.24$ \\
\hline 27 & $\$ 3,203$ & $\$ 3,502$ & $\$ 3,861$ & $\$ 4,517$ & $\$ 3,398$ & $\$ 4,280$ & $\$ 27,416.83$ \\
\hline 28 & $\$ 3,330$ & $\$ 3,640$ & $\$ 4,004$ & $\$ 4,659$ & $\$ 3,509$ & $\$ 4,436$ & $\$ 27,965.17$ \\
\hline 29 & $\$ 3,460$ & $\$ 3,779$ & $\$ 4,149$ & $\$ 4,803$ & $\$ 3,621$ & $\$ 4,596$ & $\$ 28,524.47$ \\
\hline 30 & $\$ 3,593$ & $\$ 3,922$ & $\$ 4,297$ & $\$ 4,950$ & $\$ 3,735$ & $\$ 4,758$ & $\$ 29,094.96$ \\
\hline 31 & $\$ 3,728$ & $\$ 4,067$ & $\$ 4,449$ & $\$ 5,100$ & $\$ 3,852$ & $\$ 4,924$ & $\$ 29,676.86$ \\
\hline 32 & $\$ 3,867$ & $\$ 4,216$ & $\$ 4,603$ & $\$ 5,254$ & $\$ 3,971$ & $\$ 5,094$ & $\$ 30,270.40$ \\
\hline 33 & $\$ 3,996$ & $\$ 4,367$ & $\$ 4,760$ & $\$ 5,410$ & $\$ 4,092$ & $\$ 5,266$ & $\$ 30,875.80$ \\
\hline 34 & $\$ 4,119$ & $\$ 4,522$ & $\$ 4,921$ & $\$ 5,579$ & $\$ 4,216$ & $\$ 5,443$ & $\$ 31,493.32$ \\
\hline 35 & $\$ 4,246$ & $\$ 4,679$ & $\$ 5,085$ & $\$ 5,754$ & $\$ 4,342$ & $\$ 5,622$ & $\$ 32,123.19$ \\
\hline 36 & $\$ 4,375$ & $\$ 4,840$ & $\$ 5,252$ & $\$ 5,932$ & $\$ 4,471$ & $\$ 5,806$ & $\$ 32,765.65$ \\
\hline 37 & $\$ 4,506$ & $\$ 5,003$ & $\$ 5,422$ & $\$ 6,114$ & $\$ 4,602$ & $\$ 5,992$ & $\$ 33,420.96$ \\
\hline 38 & $\$ 4,640$ & $\$ 5,171$ & $\$ 5,596$ & $\$ 6,299$ & $\$ 4,736$ & $\$ 6,183$ & $\$ 34,089.38$ \\
\hline 39 & $\$ 4,777$ & $\$ 5,341$ & $\$ 5,773$ & $\$ 6,488$ & $\$ 4,873$ & $\$ 6,378$ & $\$ 34,771.17$ \\
\hline 40 & $\$ 4,916$ & $\$ 5,515$ & $\$ 5,954$ & $\$ 6,681$ & $\$ 5,012$ & $\$ 6,576$ & $\$ 35,466.59$ \\
\hline 41 & $\$ 5,059$ & $\$ 5,692$ & $\$ 6,138$ & $\$ 6,878$ & $\$ 5,155$ & $\$ 6,778$ & $\$ 36,175.92$ \\
\hline 42 & $\$ 5,204$ & $\$ 5,873$ & $\$ 6,327$ & $\$ 7,079$ & $\$ 5,300$ & $\$ 6,985$ & $\$ 36,899.44$ \\
\hline 43 & $\$ 5,368$ & $\$ 6,058$ & $\$ 6,518$ & $\$ 7,284$ & $\$ 5,448$ & $\$ 7,195$ & $\$ 37,637.43$ \\
\hline 44 & $\$ 5,539$ & $\$ 6,246$ & $\$ 6,714$ & $\$ 7,493$ & $\$ 5,599$ & $\$ 7,410$ & $\$ 38,390.18$ \\
\hline 45 & $\$ 5,713$ & $\$ 6,438$ & $\$ 6,914$ & $\$ 7,706$ & $\$ 5,758$ & $\$ 7,629$ & $\$ 39,157.98$ \\
\hline 46 & $\$ 5,891$ & $\$ 6,634$ & $\$ 7,117$ & $\$ 7,923$ & $\$ 5,947$ & $\$ 7,853$ & $\$ 39,941.14$ \\
\hline 47 & $\$ 6,073$ & $\$ 6,833$ & $\$ 7,325$ & $\$ 8,145$ & $\$ 6,140$ & $\$ 8,106$ & $\$ 40,739.97$ \\
\hline 48 & $\$ 6,258$ & $\$ 7,037$ & $\$ 7,537$ & $\$ 8,371$ & $\$ 6,337$ & $\$ 8,371$ & $\$ 41,554.77$ \\
\hline 49 & $\$ 6,446$ & $\$ 7,245$ & $\$ 7,759$ & $\$ 8,601$ & $\$ 6,538$ & $\$ 8,641$ & $\$ 42,385.86$ \\
\hline 50 & $\$ 6,675$ & $\$ 7,493$ & $\$ 8,033$ & $\$ 8,873$ & $\$ 6,779$ & $\$ 8,947$ & $\$ 43,233.58$ \\
\hline 51 & $\$ 6,932$ & $\$ 7,770$ & $\$ 8,336$ & $\$ 9,174$ & $\$ 7,049$ & $\$ 9,279$ & $\$ 44,098.25$ \\
\hline 52 & $\$ 7,194$ & $\$ 8,052$ & $\$ 8,645$ & $\$ 9,480$ & $\$ 7,323$ & $\$ 9,618$ & $\$ 44,980.21$ \\
\hline 53 & $\$ 7,461$ & $\$ 8,340$ & $\$ 8,960$ & $\$ 9,793$ & $\$ 7,603$ & $\$ 9,963$ & $\$ 45,879.82$ \\
\hline 54 & $\$ 7,734$ & $\$ 8,634$ & $\$ 9,281$ & $\$ 10,112$ & $\$ 7,889$ & $\$ 10,315$ & $\$ 46,797.42$ \\
\hline 55 & $\$ 8,012$ & $\$ 8,933$ & $\$ 9,608$ & $\$ 10,437$ & $\$ 8,181$ & $\$ 10,674$ & $\$ 47,733.36$ \\
\hline
\end{tabular}


$\underline{\text { (Table } 3 \text { continued) }}$

\begin{tabular}{|c|c|c|c|c|c|c|c|}
\hline Age & B.C. & Alberta & Sask. & Manitoba & Ontario & Quebec & Annual Income \\
\hline 56 & $\$ 8,296$ & $\$ 9,239$ & $\$ 9,943$ & $\$ 10,769$ & $\$ 8,478$ & $\$ 11,041$ & $\$ 48,688.03$ \\
\hline 57 & $\$ 8,585$ & $\$ 9,550$ & $\$ 10,283$ & $\$ 11,107$ & $\$ 8,782$ & $\$ 11,414$ & $\$ 49,661.79$ \\
\hline 58 & $\$ 8,880$ & $\$ 9,868$ & $\$ 10,631$ & $\$ 11,453$ & $\$ 9,091$ & $\$ 11,795$ & $\$ 50,655.03$ \\
\hline 59 & $\$ 9,181$ & $\$ 10,192$ & $\$ 10,986$ & $\$ 11,805$ & $\$ 9,407$ & $\$ 12,184$ & $\$ 51,668.13$ \\
\hline 60 & $\$ 9,487$ & $\$ 10,523$ & $\$ 11,347$ & $\$ 12,163$ & $\$ 9,728$ & $\$ 12,580$ & $\$ 52,701.49$ \\
\hline 61 & $\$ 9,801$ & $\$ 10,860$ & $\$ 11,716$ & $\$ 12,530$ & $\$ 10,057$ & $\$ 12,985$ & $\$ 53,755.52$ \\
\hline 62 & $\$ 10,120$ & $\$ 11,204$ & $\$ 12,092$ & $\$ 12,903$ & $\$ 10,392$ & $\$ 13,397$ & $\$ 54,830.63$ \\
\hline 63 & $\$ 10,446$ & $\$ 11,555$ & $\$ 12,476$ & $\$ 13,285$ & $\$ 10,733$ & $\$ 13,818$ & $\$ 55,927.24$ \\
\hline 64 & $\$ 10,778$ & $\$ 11,913$ & $\$ 12,868$ & $\$ 13,673$ & $\$ 11,082$ & $\$ 14,247$ & $\$ 57,045.79$ \\
\hline 65 & $\$ 11,117$ & $\$ 12,278$ & $\$ 13,267$ & $\$ 14,070$ & $\$ 11,437$ & $\$ 14,685$ & $\$ 58,186.70$ \\
\hline 66 & $\$ 11,462$ & $\$ 12,651$ & $\$ 13,674$ & $\$ 14,474$ & $\$ 11,800$ & $\$ 15,132$ & $\$ 59,350.44$ \\
\hline 67 & $\$ 11,815$ & $\$ 13,031$ & $\$ 14,090$ & $\$ 14,886$ & $\$ 12,169$ & $\$ 15,587$ & $\$ 60,537.45$ \\
\hline 68 & $\$ 0.00$ & $\$ 0.00$ & $\$ 0.00$ & $\$ 0.00$ & $\$ 0.00$ & $\$ 0.00$ & $\$ 0.00$ \\
\hline 69 & $\$ 0.00$ & $\$ 0.00$ & $\$ 0.00$ & $\$ 0.00$ & $\$ 0.00$ & $\$ 0.00$ & $\$ 0.00$ \\
\hline 70 & $\$ 0.00$ & $\$ 0.00$ & $\$ 0.00$ & $\$ 0.00$ & $\$ 0.00$ & $\$ 0.00$ & $\$ 0.00$ \\
\hline 71 & $\$ 0.00$ & $\$ 0.00$ & $\$ 0.00$ & $\$ 0.00$ & $\$ 0.00$ & $\$ 0.00$ & $\$ 0.00$ \\
\hline 72 & $\$ 0.00$ & $\$ 0.00$ & $\$ 0.00$ & $\$ 0.00$ & $\$ 0.00$ & $\$ 0.00$ & $\$ 0.00$ \\
\hline 73 & $\$ 0.00$ & $\$ 0.00$ & $\$ 0.00$ & $\$ 0.00$ & $\$ 0.00$ & $\$ 0.00$ & $\$ 0.00$ \\
\hline 74 & $\$ 0.00$ & $\$ 0.00$ & $\$ 0.00$ & $\$ 0.00$ & $\$ 0.00$ & $\$ 0.00$ & $\$ 0.00$ \\
\hline 75 & $\$ 0.00$ & $\$ 0.00$ & $\$ 0.00$ & $\$ 0.00$ & $\$ 0.00$ & $\$ 0.00$ & $\$ 0.00$ \\
\hline 76 & $\$ \mathbf{\$ 0 . 0 0}$ & $\$ 0.00$ & $\$ 0.00$ & $\$ 0.00$ & $\$ 0.00$ & $\$ 0.00$ & $\$ 0.00$ \\
\hline 77 & $\$ 0.00$ & $\$ 0.00$ & $\$ 0.00$ & $\$ 0.00$ & $\$ 0.00$ & $\$ 0.00$ & $\$ 0.00$ \\
\hline 78 & $\$ 0.00$ & $\$ 0.00$ & $\$ 0.00$ & $\$ 0.00$ & $\$ 0.00$ & $\$ 0.00$ & $\$ 0.00$ \\
\hline 79 & $\$ 0.00$ & $\$ 0.00$ & $\$ 0.00$ & $\$ 0.00$ & $\$ 0.00$ & $\$ 0.00$ & $\$ 0.00$ \\
\hline 80 & $\$ 0.00$ & $\$ 0.00$ & $\$ 0.00$ & $\$ 0.00$ & $\$ 0.00$ & $\$ 0.00$ & $\$ 0.00$ \\
\hline Total & $\$ 293,604$ & $\$ 326,101$ & $\$ 352,792$ & $\$ 389,376$ & $\$ 302,112$ & $\$ 390,948$ & $\$ 1,917,409$ \\
\hline
\end{tabular}

Table 4. Lifetime Taxes Paid by a Citizen Starting at $\$ 11.25$ / hour

and a $2 \%$ Annual Increase in the Canadian Provinces of N.B, N.S., P.E.I., Nfld., N.W.T., Yukon, \& Nunavut

Lifetime Taxes Paid by a Citizen Starting at $\$ 11.25$ / hour and a 2\% Annual Increase in the Canadian Provinces of N.B, N.S., P.E.I., Nfld., N.W.T., Yukon, \& Nunavut.

\begin{tabular}{|c|c|c|c|c|c|c|c|c|}
\hline Age & N.B. & N.S. & P.E.I. & Nfld. & N.W.T. & Yukon & Nunavut & Annual Income \\
\hline 1 & $\$ 0.00$ & $\$ 0.00$ & $\$ \mathbf{\$ 0 . 0 0}$ & $\$ 0.00$ & $\$ 0.00$ & $\$ 0.00$ & $\$ 0.00$ & $\$ \mathbf{\$ 0 . 0 0}$ \\
\hline 2 & $\$ \mathbf{\$ 0 . 0 0}$ & $\$ \mathbf{\$ 0 . 0 0}$ & $\$ 0.00$ & $\$ 0.00$ & $\$ 0.00$ & $\$ 0.00$ & $\$ 0.00$ & $\$ \mathbf{\$ 0 . 0 0}$ \\
\hline 3 & $\$ 0.00$ & $\$ \mathbf{\$ 0 . 0 0}$ & $\$ 0.00$ & $\$ 0.00$ & $\$ 0.00$ & $\$ \mathbf{\$ 0 . 0 0}$ & $\$ 0.00$ & $\$ 0.00$ \\
\hline 4 & $\$ 0.00$ & $\$ 0.00$ & $\$ 0.00$ & $\$ 0.00$ & $\$ 0.00$ & $\$ 0.00$ & $\$ 0.00$ & $\$ 0.00$ \\
\hline 5 & $\$ 0.00$ & $\$ 0.00$ & $\$ 0.00$ & $\$ 0.00$ & $\$ 0.00$ & $\$ 0.00$ & $\$ 0.00$ & $\$ 0.00$ \\
\hline 6 & $\$ 0.00$ & $\$ 0.00$ & $\$ 0.00$ & $\$ 0.00$ & $\$ 0.00$ & $\$ 0.00$ & $\$ 0.00$ & $\$ 0.00$ \\
\hline 7 & $\$ 0.00$ & $\$ 0.00$ & $\$ 0.00$ & $\$ 0.00$ & $\$ 0.00$ & $\$ 0.00$ & $\$ 0.00$ & $\$ 0.00$ \\
\hline 8 & $\$ 0.00$ & $\$ 0.00$ & $\$ 0.00$ & $\$ 0.00$ & $\$ 0.00$ & $\$ 0.00$ & $\$ 0.00$ & $\$ 0.00$ \\
\hline 9 & $\$ 0.00$ & $\$ 0.00$ & $\$ 0.00$ & $\$ 0.00$ & $\$ 0.00$ & $\$ 0.00$ & $\$ 0.00$ & $\$ 0.00$ \\
\hline 10 & $\$ 0.00$ & $\$ 0.00$ & $\$ 0.00$ & $\$ 0.00$ & $\$ 0.00$ & $\$ 0.00$ & $\$ 0.00$ & $\$ 0.00$ \\
\hline 11 & $\$ 0.00$ & $\$ 0.00$ & $\$ 0.00$ & $\$ 0.00$ & $\$ 0.00$ & $\$ 0.00$ & $\$ 0.00$ & $\$ 0.00$ \\
\hline 12 & $\$ 0.00$ & $\$ 0.00$ & $\$ 0.00$ & $\$ 0.00$ & $\$ 0.00$ & $\$ 0.00$ & $\$ 0.00$ & $\$ 0.00$ \\
\hline 13 & $\$ 0.00$ & $\$ 0.00$ & $\$ 0.00$ & $\$ 0.00$ & $\$ 0.00$ & $\$ 0.00$ & $\$ 0.00$ & $\$ 0.00$ \\
\hline 14 & $\$ 0.00$ & $\$ \mathbf{\$ 0 . 0 0}$ & $\$ 0.00$ & $\$ 0.00$ & $\$ 0.00$ & $\$ 0.00$ & $\$ 0.00$ & $\$ 0.00$ \\
\hline 15 & $\$ 0.00$ & $\$ 0.00$ & $\$ 0.00$ & $\$ 0.00$ & $\$ 0.00$ & $\$ 0.00$ & $\$ 0.00$ & $\$ 0.00$ \\
\hline 16 & $\$ 0.00$ & $\$ 0.00$ & $\$ 0.00$ & $\$ 0.00$ & $\$ 0.00$ & $\$ 0.00$ & $\$ 0.00$ & $\$ 0.00$ \\
\hline 17 & $\$ 0.00$ & $\$ 0.00$ & $\$ 0.00$ & $\$ 0.00$ & $\$ 0.00$ & $\$ 0.00$ & $\$ 0.00$ & $\$ 0.00$ \\
\hline 18 & $\$ 0.00$ & $\$ 0.00$ & $\$ 0.00$ & $\$ 0.00$ & $\$ \mathbf{\$ 0 . 0 0}$ & $\$ 0.00$ & $\$ 0.00$ & $\mathbf{\$ 0 . 0 0}$ \\
\hline 19 & $\$ 2,859$ & $\$ 3,198$ & $\$ 3,424$ & $\$ 2,980$ & $\$ 2,484$ & $\$ 2,772$ & $\$ 2,334$ & $\$ 23,400.00$ \\
\hline 20 & $\$ 2,986$ & $\$ 3,309$ & $\$ 3,540$ & $\$ 3,086$ & $\$ 2,581$ & $\$ 2,875$ & $\$ 2,423$ & $\$ 23,868.00$ \\
\hline 21 & $\$ 3,115$ & $\$ 3,423$ & $\$ 3,659$ & $\$ 3,194$ & $\$ 2,681$ & $\$ 2,980$ & $\$ 2,514$ & $\$ 24,345.36$ \\
\hline 22 & $\$ 3,247$ & $\$ 3,539$ & $\$ 3,779$ & $\$ 3,305$ & $\$ 2,783$ & $\$ 3,088$ & $\$ 2,606$ & $\$ 24,832.27$ \\
\hline
\end{tabular}


$\underline{\text { (Table } 4 \text { continued) }}$

\begin{tabular}{|c|c|c|c|c|c|c|c|c|}
\hline Age & N.B. & N.S. & P.E.I. & Nfld. & N.W.T. & Yukon & Nunavut & Annual Income \\
\hline 23 & $\$ 3,382$ & $\$ 3,657$ & $\$ 3,902$ & $\$ 3,418$ & $\$ 2,887$ & $\$ 3,197$ & $\$ 2,700$ & $\$ 25,328.91$ \\
\hline 24 & $\$ 3,519$ & $\$ 3,777$ & $\$ 4,028$ & $\$ 3,533$ & $\$ 2,992$ & $\$ 3,309$ & $\$ 2,797$ & $\$ 25,835.49$ \\
\hline 25 & $\$ 3,659$ & $\$ 3,900$ & $\$ 4,156$ & $\$ 3,650$ & $\$ 3,101$ & $\$ 3,423$ & $\$ 2,895$ & $\$ 26,352.20$ \\
\hline 26 & $\$ 3,802$ & $\$ 4,025$ & $\$ 4,287$ & $\$ 3,770$ & $\$ 3,211$ & $\$ 3,539$ & $\$ 2,995$ & $\$ 26,879.24$ \\
\hline 27 & $\$ 3,948$ & $\$ 4,153$ & $\$ 4,420$ & $\$ 3,892$ & $\$ 3,323$ & $\$ 3,657$ & $\$ 3,097$ & $\$ 27,416.83$ \\
\hline 28 & $\$ 4,097$ & $\$ 4,284$ & $\$ 4,556$ & $\$ 4,016$ & $\$ 3,438$ & $\$ 3,778$ & $\$ 3,201$ & $\$ 27,965.17$ \\
\hline 29 & $\$ 4,248$ & $\$ 4,417$ & $\$ 4,695$ & $\$ 4,143$ & $\$ 3,554$ & $\$ 3,901$ & $\$ 3,308$ & $\$ 28,524.47$ \\
\hline 30 & $\$ 4,402$ & $\$ 4,552$ & $\$ 4,836$ & $\$ 4,272$ & $\$ 3,674$ & $\$ 4,027$ & $\$ 3,416$ & $\$ 29,094.96$ \\
\hline 31 & $\$ 4,560$ & $\$ 4,696$ & $\$ 4,981$ & $\$ 4,405$ & $\$ 3,795$ & $\$ 4,155$ & $\$ 3,527$ & $\$ 29,676.86$ \\
\hline 32 & $\$ 4,721$ & $\$ 4,874$ & $\$ 5,128$ & $\$ 4,539$ & $\$ 3,919$ & $\$ 4,286$ & $\$ 3,639$ & $\$ 30,270.40$ \\
\hline 33 & $\$ 4,885$ & $\$ 5,055$ & $\$ 5,278$ & $\$ 4,677$ & $\$ 4,046$ & $\$ 4,419$ & $\$ 3,754$ & $\$ 30,875.80$ \\
\hline 34 & $\$ 5,053$ & $\$ 5,240$ & $\$ 5,431$ & $\$ 4,817$ & $\$ 4,175$ & $\$ 4,556$ & $\$ 3,872$ & $\$ 31,493.32$ \\
\hline 35 & $\$ 5,223$ & $\$ 5,429$ & $\$ 5,593$ & $\$ 4,960$ & $\$ 4,307$ & $\$ 4,695$ & $\$ 3,991$ & $\$ 32,123.19$ \\
\hline 36 & $\$ 5,397$ & $\$ 5,621$ & $\$ 5,778$ & $\$ 5,106$ & $\$ 4,441$ & $\$ 4,836$ & $\$ 4,113$ & $\$ 32,765.65$ \\
\hline 37 & $\$ 5,575$ & $\$ 5,817$ & $\$ 5,967$ & $\$ 5,280$ & $\$ 4,578$ & $\$ 4,980$ & $\$ 4,238$ & $\$ 33,420.96$ \\
\hline 38 & $\$ 5,754$ & $\$ 6,018$ & $\$ 6,159$ & $\$ 5,464$ & $\$ 4,718$ & $\$ 5,128$ & $\$ 4,365$ & $\$ 34,089.38$ \\
\hline 39 & $\$ 5,919$ & $\$ 6,222$ & $\$ 6,356$ & $\$ 5,651$ & $\$ 4,860$ & $\$ 5,278$ & $\$ 4,495$ & $\$ 34,771.17$ \\
\hline 40 & $\$ 6,086$ & $\$ 6,430$ & $\$ 6,556$ & $\$ 5,842$ & $\$ 5,005$ & $\$ 5,431$ & $\$ 4,627$ & $\$ 35,466.59$ \\
\hline 41 & $\$ 6,257$ & $\$ 6,643$ & $\$ 6,760$ & $\$ 6,037$ & $\$ 5,154$ & $\$ 5,588$ & $\$ 4,761$ & $\$ 36,175.92$ \\
\hline 42 & $\$ 6,432$ & $\$ 6,859$ & $\$ 6,969$ & $\$ 6,236$ & $\$ 5,305$ & $\$ 5,747$ & $\$ 4,899$ & $\$ 36,899.44$ \\
\hline 43 & $\$ 6,610$ & $\$ 7,080$ & $\$ 7,181$ & $\$ 6,439$ & $\$ 5,459$ & $\$ 5,910$ & $\$ 5,039$ & $\$ 37,637.43$ \\
\hline 44 & $\$ 6,797$ & $\$ 7,306$ & $\$ 7,398$ & $\$ 6,646$ & $\$ 5,616$ & $\$ 6,076$ & $\$ 5,182$ & $\$ 38,390.18$ \\
\hline 45 & $\$ 7,005$ & $\$ 7,536$ & $\$ 7,619$ & $\$ 6,857$ & $\$ 5,790$ & $\$ 6,245$ & $\$ 5,328$ & $\$ 39,157.98$ \\
\hline 46 & $\$ 7,217$ & $\$ 7,771$ & $\$ 7,845$ & $\$ 7,073$ & $\$ 5,975$ & $\$ 6,418$ & $\$ 5,477$ & $\$ 39,941.14$ \\
\hline 47 & $\$ 7,434$ & $\$ 8,010$ & $\$ 8,075$ & $\$ 7,292$ & $\$ 6,163$ & $\$ 6,593$ & $\$ 5,629$ & $\$ 40,739.97$ \\
\hline 48 & $\$ 7,654$ & $\$ 8,254$ & $\$ 8,309$ & $\$ 7,517$ & $\$ 6,355$ & $\$ 6,773$ & $\$ 5,808$ & $\$ 41,554.77$ \\
\hline 49 & $\$ 7,880$ & $\$ 8,502$ & $\$ 8,549$ & $\$ 7,745$ & $\$ 6,551$ & $\$ 6,956$ & $\$ 5,991$ & $\$ 42,385.86$ \\
\hline 50 & $\$ 8,146$ & $\$ 8,793$ & $\$ 8,830$ & $\$ 8,015$ & $\$ 6,788$ & $\$ 7,194$ & $\$ 6,214$ & $\$ 43,233.58$ \\
\hline 51 & $\$ 8,441$ & $\$ 9,113$ & $\$ 9,139$ & $\$ 8,313$ & $\$ 7,053$ & $\$ 7,468$ & $\$ 6,465$ & $\$ 44,098.25$ \\
\hline 52 & $\$ 8,742$ & $\$ 9,439$ & $\$ 9,455$ & $\$ 8,618$ & $\$ 7,323$ & $\$ 7,747$ & $\$ 6,721$ & $\$ 44,980.21$ \\
\hline 53 & $\$ 9,048$ & $\$ 9,771$ & $\$ 9,777$ & $\$ 8,928$ & $\$ 7,598$ & $\$ 8,032$ & $\$ 6,982$ & $\$ 45,879.82$ \\
\hline 54 & $\$ 9,361$ & $\$ 10,110$ & $\$ 10,105$ & $\$ 9,245$ & $\$ 7,879$ & $\$ 8,323$ & $\$ 7,248$ & $\$ 46,797.42$ \\
\hline 55 & $\$ 9,681$ & $\$ 10,456$ & $\$ 10,441$ & $\$ 9,568$ & $\$ 8,165$ & $\$ 8,619$ & $\$ 7,519$ & $\$ 47,733.36$ \\
\hline 56 & $\$ 10,006$ & $\$ 10,809$ & $\$ 10,782$ & $\$ 9,897$ & $\$ 8,458$ & $\$ 8,922$ & $\$ 7,796$ & $\$ 48,688.03$ \\
\hline 57 & $\$ 10,338$ & $\$ 11,168$ & $\$ 11,131$ & $\$ 10,233$ & $\$ 8,755$ & $\$ 9,230$ & $\$ 8,079$ & $\$ 49,661.79$ \\
\hline 58 & $\$ 10,677$ & $\$ 11,536$ & $\$ 11,487$ & $\$ 10,576$ & $\$ 9,059$ & $\$ 9,545$ & $\$ 8,367$ & $\$ 50,655.03$ \\
\hline 59 & $\$ 11,022$ & $\$ 11,910$ & $\$ 11,849$ & $\$ 10,925$ & $\$ 9,369$ & $\$ 9,866$ & $\$ 8,661$ & $\$ 51,668.13$ \\
\hline 60 & $\$ 11,375$ & $\$ 12,292$ & $\$ 12,219$ & $\$ 11,281$ & $\$ 9,686$ & $\$ 10,193$ & $\$ 8,960$ & $\$ 52,701.49$ \\
\hline 61 & $\$ 11,734$ & $\$ 12,681$ & $\$ 12,596$ & $\$ 11,645$ & $\$ 10,008$ & $\$ 10,527$ & $\$ 9,266$ & $\$ 53,755.52$ \\
\hline 62 & $\$ 12,101$ & $\$ 13,078$ & $\$ 12,981$ & $\$ 12,016$ & $\$ 10,337$ & $\$ 10,868$ & $\$ 9,578$ & $\$ 54,830.63$ \\
\hline 63 & $\$ 12,475$ & $\$ 13,484$ & $\$ 13,374$ & $\$ 12,394$ & $\$ 10,673$ & $\$ 11,215$ & $\$ 9,896$ & $\$ 55,927.24$ \\
\hline 64 & $\$ 12,856$ & $\$ 13,897$ & $\$ 13,774$ & $\$ 12,780$ & $\$ 11,015$ & $\$ 11,569$ & $\$ 10,220$ & $\$ 57,045.79$ \\
\hline 65 & $\$ 13,245$ & $\$ 14,318$ & $\$ 14,183$ & $\$ 13,174$ & $\$ 11,364$ & $\$ 11,931$ & $\$ 10,551$ & $\$ 58,186.70$ \\
\hline 66 & $\$ 13,642$ & $\$ 14,751$ & $\$ 14,599$ & $\$ 13,575$ & $\$ 11,720$ & $\$ 12,300$ & $\$ 10,888$ & $\$ 59,350.44$ \\
\hline 67 & $\$ 14,047$ & $\$ 15,210$ & $\$ 15,024$ & $\$ 13,985$ & $\$ 12,083$ & $\$ 12,676$ & $\$ 11,233$ & $\$ 60,537.45$ \\
\hline 68 & $\$ 0.00$ & $\$ 0.00$ & $\$ 0.00$ & $\$ 0.00$ & $\$ 0.00$ & $\$ 0.00$ & $\$ 0.00$ & $\$ 0.00$ \\
\hline 69 & $\$ 0.00$ & $\$ 0.00$ & $\$ 0.00$ & $\$ 0.00$ & $\$ 0.00$ & $\$ 0.00$ & $\$ 0.00$ & $\$ 0.00$ \\
\hline 70 & $\$ 0.00$ & $\$ 0.00$ & $\$ 0.00$ & $\$ 0.00$ & $\$ 0.00$ & $\$ 0.00$ & $\$ 0.00$ & $\$ 0.00$ \\
\hline 71 & $\$ 0.00$ & $\$ 0.00$ & $\$ 0.00$ & $\$ 0.00$ & $\$ 0.00$ & $\$ 0.00$ & $\$ 0.00$ & $\$ 0.00$ \\
\hline 72 & $\$ 0.00$ & $\$ 0.00$ & $\$ 0.00$ & $\$ 0.00$ & $\$ 0.00$ & $\$ 0.00$ & $\$ 0.00$ & $\$ 0.00$ \\
\hline 73 & $\$ 0.00$ & $\$ 0.00$ & $\$ 0.00$ & $\$ 0.00$ & $\$ \mathbf{\$ 0 . 0 0}$ & $\$ 0.00$ & $\$ 0.00$ & $\$ 0.00$ \\
\hline 74 & $\$ 0.00$ & $\$ 0.00$ & $\$ 0.00$ & $\$ 0.00$ & $\$ 0.00$ & $\$ 0.00$ & $\$ 0.00$ & $\$ 0.00$ \\
\hline 75 & $\$ 0.00$ & $\$ 0.00$ & $\$ 0.00$ & $\$ 0.00$ & $\$ 0.00$ & $\$ 0.00$ & $\$ 0.00$ & $\$ 0.00$ \\
\hline 76 & $\$ 0.00$ & $\$ 0.00$ & $\$ 0.00$ & $\$ 0.00$ & $\$ 0.00$ & $\$ 0.00$ & $\$ 0.00$ & $\$ 0.00$ \\
\hline 77 & $\$ 0.00$ & $\$ 0.00$ & $\$ 0.00$ & $\$ 0.00$ & $\$ 0.00$ & $\$ 0.00$ & $\$ 0.00$ & $\$ 0.00$ \\
\hline 78 & $\$ 0.00$ & $\$ 0.00$ & $\$ 0.00$ & $\$ 0.00$ & $\$ 0.00$ & $\$ 0.00$ & $\$ 0.00$ & $\$ 0.00$ \\
\hline 79 & $\$ 0.00$ & $\$ 0.00$ & $\$ 0.00$ & $\$ 0.00$ & $\$ 0.00$ & $\$ 0.00$ & $\$ 0.00$ & $\$ 0.00$ \\
\hline 80 & $\$ \mathbf{\$ 0 . 0 0}$ & $\$ \mathbf{\$ 0 . 0 0}$ & $\$ \mathbf{\$ 0 . 0 0}$ & $\$ \mathbf{\$ 0 . 0 0}$ & $\$ 0.00$ & $\$ 0.00$ & $\$ \mathbf{\$ 0 . 0 0}$ & $\$ \mathbf{\$ 0 . 0 0}$ \\
\hline Total & $\$ 356,660$ & $\$ 382,413$ & $\$ 386,960$ & $\$ 351,010$ & $\$ 300,254$ & $\$ 320,841$ & $\$ 277,665$ & $\$ 1,917,409$ \\
\hline
\end{tabular}


Table 5 (below) is a summary of the information provided for all Canadian provinces and territories' lifetime income tax comparison of two hypothetical citizens who have a one-dollar per hour difference in their earnings. The table also contains the mean and the standard deviation of the compared variables and also the lifetime variance of income tax contributions for these two hypothetical citizens. The national average of extra taxes (variance) paid by the higher earning hypothetical citizen is $\$ \underline{48,301}$ and the province of Quebec has the highest variance for the difference between the earnings of these two citizens $(\$ 56,368)$ and Nunavut has the lowest variance in earnings $(\$ 40,033)$. Figure 1 shows the same results in a chart format.

Table 5. Comparing Lifetime Taxes Paid by Two Hypothetical Citizens, One Starting at $\$ 11.25 / \mathrm{hr}$. and One at $\$ 10.25 / \mathrm{hr}$. (all Canadian provinces and territories).

\begin{tabular}{|c|c|c|c|}
\hline \multicolumn{4}{|c|}{ Comparing Lifetime Taxes Paid by Two Citizen, One Staring at $\$ 11.25$ and One at $\$ 10.25$} \\
\hline Province / Territory & $\begin{array}{c}\text { Lifetime Taxes Paid by a } \\
\text { Citizen Starting at } \$ 11.25 / \text { hou } \\
\text { and } 2 \% \text { annual increase }\end{array}$ & $\begin{array}{l}\text { Lifetime Taxes Paid by a Citizen } \\
\text { IrStarting at } \$ 10.25 \text { / hour and } 2 \% \\
\text { annual increase }\end{array}$ & $\begin{array}{c}\text { Extra Taxes Received by a } \\
\text { Provincial Gov. Due to a Higher } \\
\text { Minimum Wage }\end{array}$ \\
\hline B.C. & $\$ 293,604.00$ & $\$ 250,341.00$ & $\$ 43,263.00$ \\
\hline Alberta & $\$ 326,101.00$ & $\$ 278,335.00$ & $\$ 47,766.00$ \\
\hline Sask. & $\$ 352,792.00$ & $\$ 301,786.00$ & $\$ 51,006.00$ \\
\hline Manitoba & $\$ 389,376.00$ & $\$ 337,732.00$ & $\$ 51,644.00$ \\
\hline Ontario & $\$ 302,112.00$ & $\$ 259,050.00$ & $\$ 43,062.00$ \\
\hline Quebec & $\$ 390,948.00$ & $\$ 334,580.00$ & $\$ 56,368.00$ \\
\hline N.B. & $\$ 356,660.00$ & $\$ 305,896.00$ & $\$ 50,764.00$ \\
\hline N.S. & $\$ 382,413.00$ & $\$ 328,342.00$ & $\$ 54,071.00$ \\
\hline P.E.I. & $\$ 386,960.00$ & $\$ 334,572.00$ & $\$ 52,388.00$ \\
\hline Nfld. & $\$ 351,010.00$ & $\$ 301,406.00$ & $\$ 49,604.00$ \\
\hline N.W.T. & $\$ 300,254.00$ & $\$ 256,970.00$ & $\$ 43,284.00$ \\
\hline Yukon & $\$ 320,841.00$ & $\$ 276,169.00$ & $\$ 44,672.00$ \\
\hline Nunavut & $\$ 277,665.00$ & $\$ 237,632.00$ & $\$ 40,033.00$ \\
\hline Mean & $\$ 340,825.85$ & $\$ 292,523.92$ & $\$ 48,301.92$ \\
\hline SD & $\$ 40,005.28$ & $\$ 35,181.51$ & $\$ 5,009.53$ \\
\hline
\end{tabular}

Figure 1. Comparing Lifetime taxes paid by two hypothetical citizens, one starting at $\$ 11.25 /$ hour and one starting at $\$ 10.25$ / hour

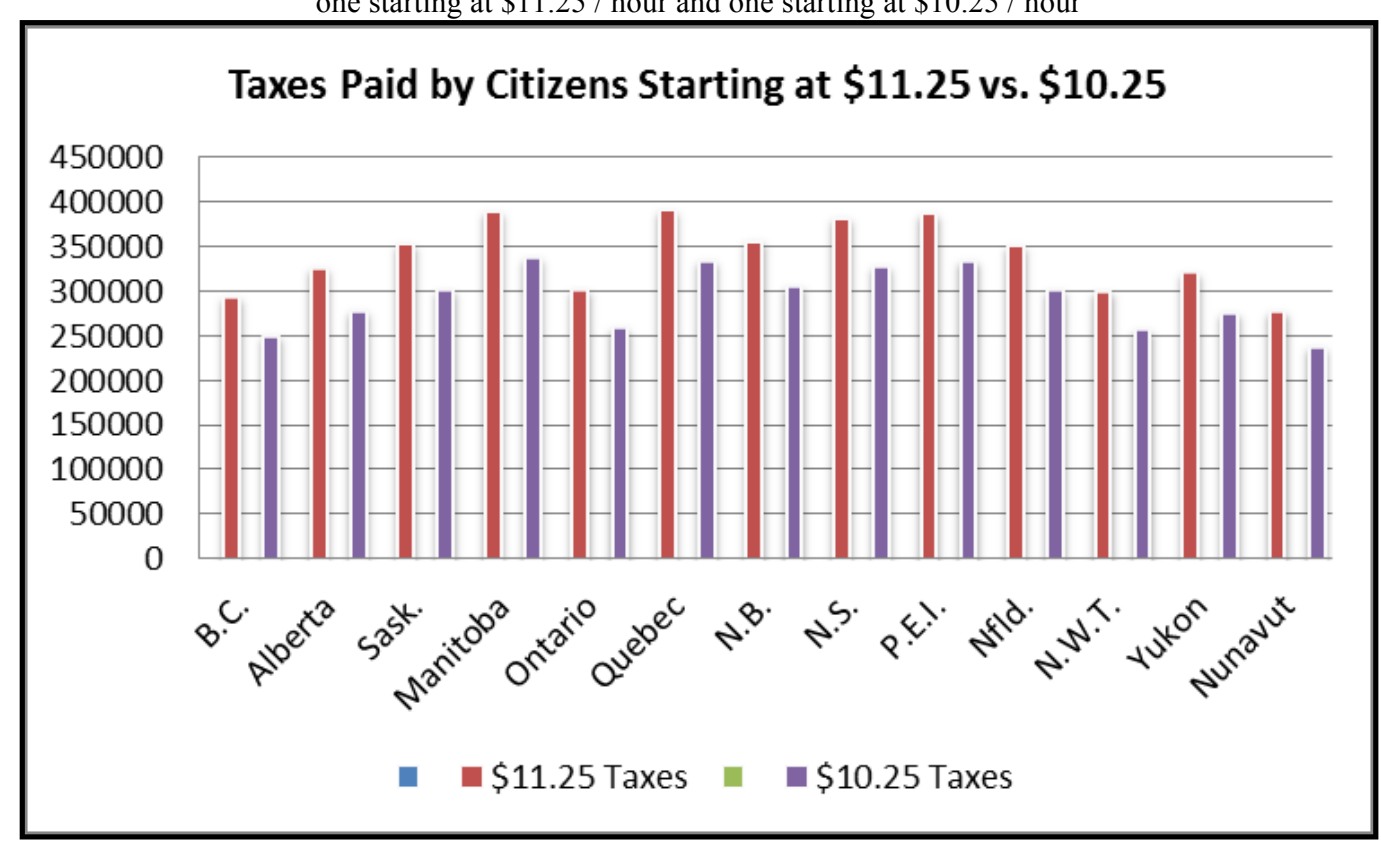




\section{DISCUSSION OF THE RESULTS}

It was surprising to discover the effect of this unexpected method to pay for human capital development through higher education and to see the large increase in lifetime income tax revenues due to ane-dollar increase in the minimum wage. When the two hypothetical citizens with a one dollar difference in their hourly wages $(\$ 10.25$ $\& \$ 11.25$ / hour) were compared, the citizen with the higher wage (\$11.25) was found to contribute a lifelong amount of $\$ 48,301$ more in income taxes due to the one dollar increase in the citizen's wage (Table 5). This means that the government income tax opportunity cost (the forgone taxes) of keeping the minimum wage at $\$ 10.25$ (or lower in some provinces) is equal to $\$ 48,301$ for every minimum wage working citizen in Canada.

Figure 2 (below) shows a chart of the tax payments of the two hypothetical citizens and their interaction with government transfer payments. The two hypothetical citizens have both entered the working stage at age 19 and worked untill they reached the Canadian retirement age of 67. The chart shows that a one dollar more in wages per hour increase in Canada can translate into an increased contribution of $\$ 48,301$ in income taxes over the lifetime of these two hypothetical citizens and a reduction of the breakeven age of 4.6 years.

Figure 2. Government-citizen funding cycle for two hypothetical citizens in Canada, one with a starting wage of $\$ 10.25$ / hour and one with a starting wage of $\$ 11.25$ / hour

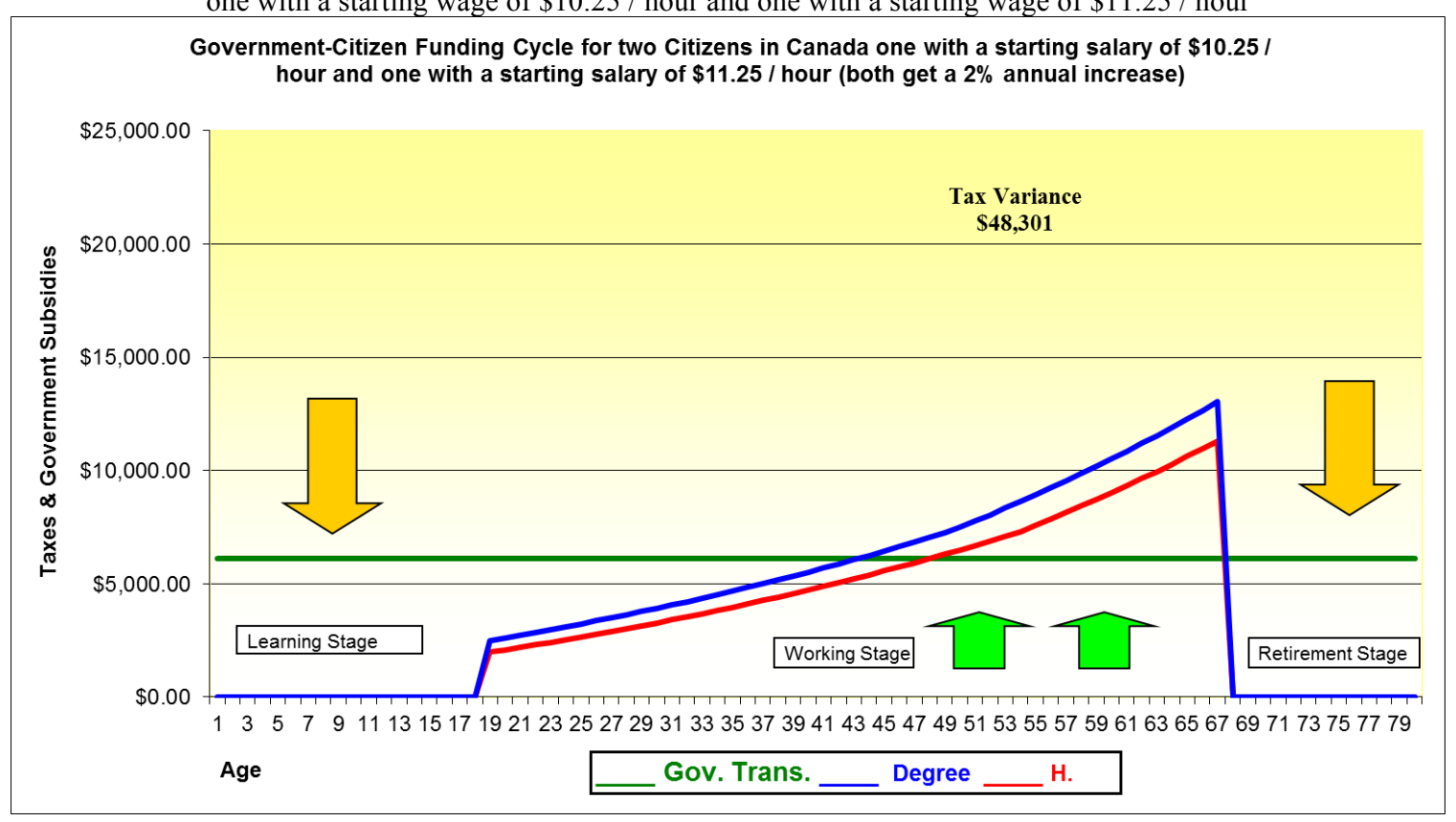

The aim of comparing these two hypothetical citizens in Figure 2 (above) is to see whether a one dollar per hour difference in a citizen's income due to extra training could generate enough tax revenue to justify, financially, the public investment in human capital development through post-secondary education. Another way to interprit the results of this comparison is to conclude that any training beyond the high school level that could facilitate any higher income (even one dollar above the minimum wage) could, in fact, justify public investment in human capital development. In other words, comparing two citizens with only one dollar difference in their earnings is used to show the financial consequences and the 'worst case scenario' for a government budget as a result of public investment in higher education.

Table 6 (below) summarizes the data from all Canadian provinces and territories of the comparison of the number of years it would take to break even for the two hypothetical citizens with a one-dollar difference in their hourly wages. Results (Table 6 below) show that the average age to break even at the national level, in the case of the $\$ 11.25 /$ hour citizen is 42.8 years, and in the case of the $\$ 10.25 / \mathrm{hr}$. citizen is 47.5 years, a gap of 4.6 years between the two compared hypothetical citizens. This means that a one-dollar increase in hourly earnings due to 
any kind of training has the potential to save 4.6 years of subsidization by the government. Figure 3 shows the same results in a chart format.

Table 6. Comparing Years to Breakeven for Two Hypothetical Citizens, One Starting at $\$ 11.25 / \mathrm{hr}$. vs. one starting at $\$ 10.25 / \mathrm{hr}$ Comparing Years to Breakeven for Two Citizen, One Staring at $\$ 11.25$ vs. $\$ 10.25$

Years to Breakeven for a Years to Breakeven for a

Province / Territory Citizen Starting at \$11.25 / hourCitizen Starting at \$10.25 / hour Variance in Years to Breakeven

and $2 \%$ annual increase and $2 \%$ annual increase

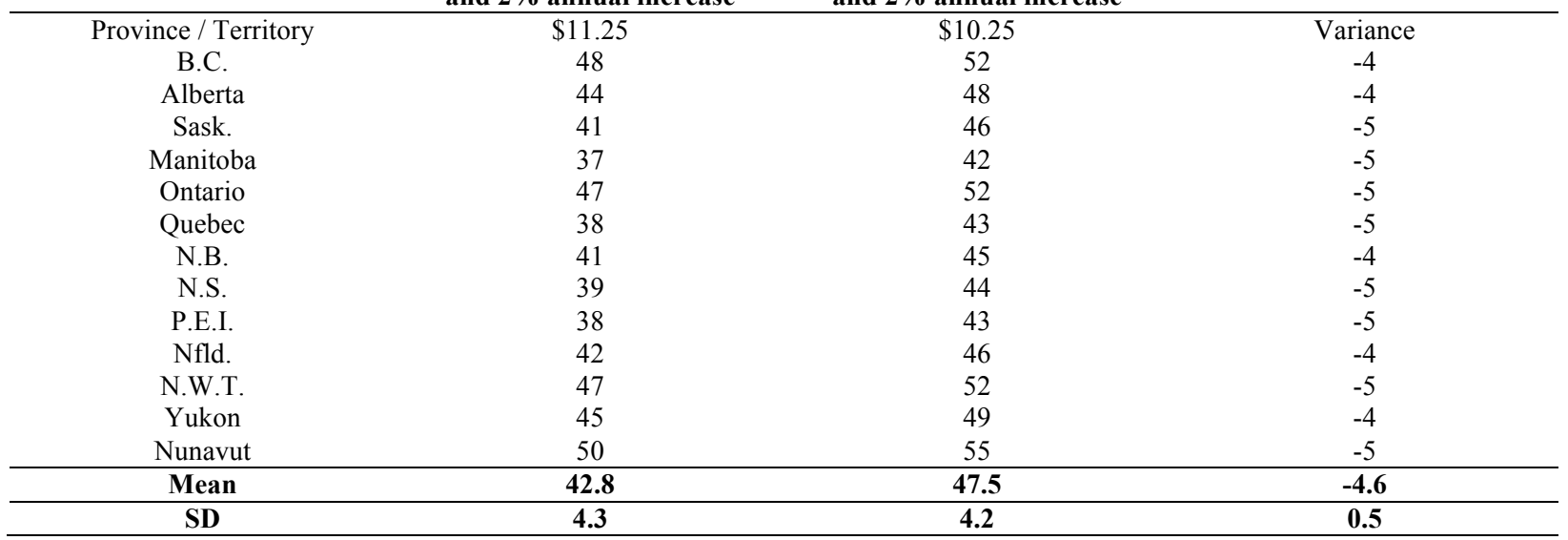

Figure 3. Years to Breakeven for hypothetical citizens starting at $\$ 11.25 /$ hour and hypothetical citizens starting at $\$ 10.25 /$ hour

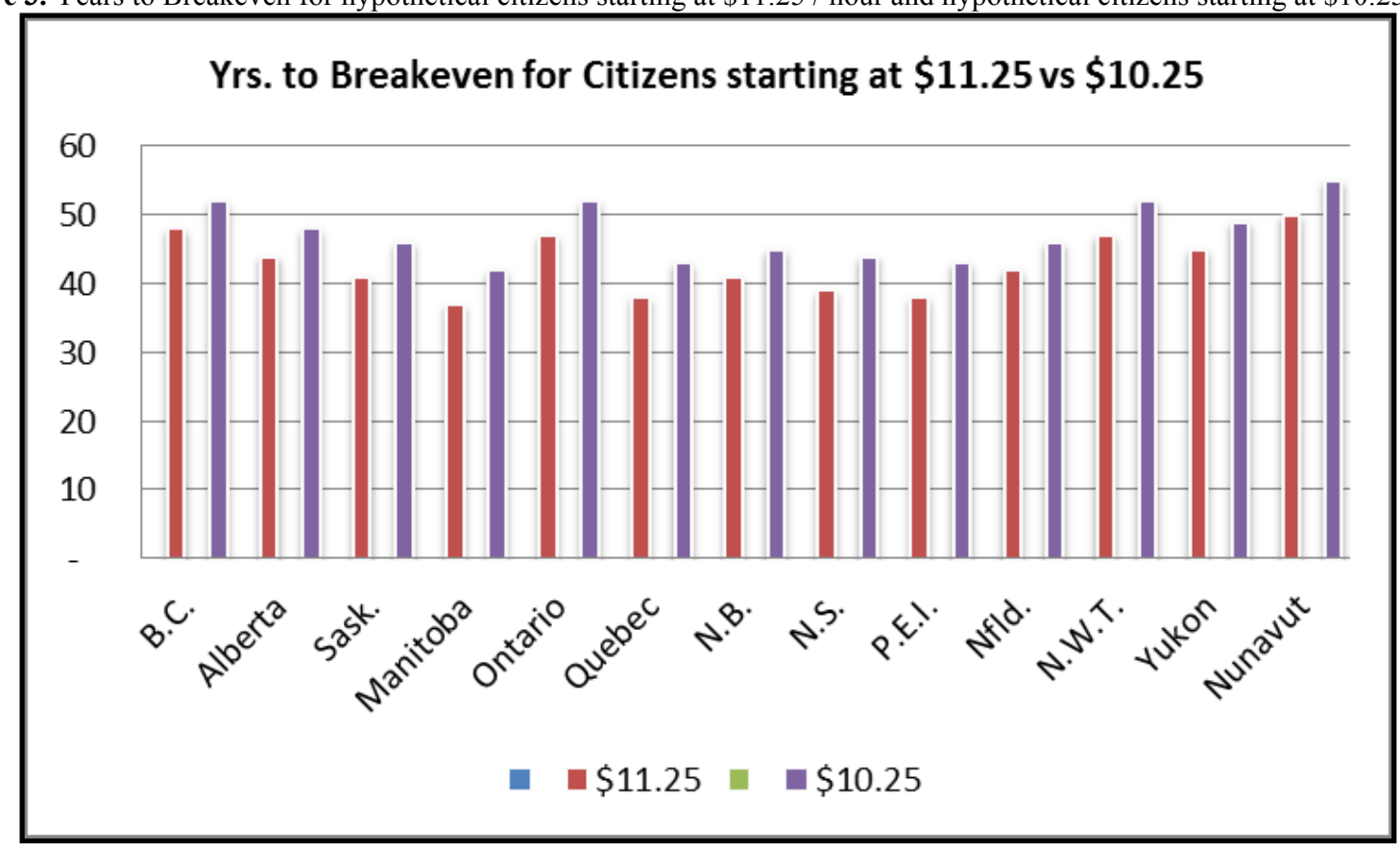

Also, while this experiment demonstrated the effect of a one-dollar increase in the minimum wage on income taxes, it could be argued that other taxes (e. g., sales taxes) are also expected to increase due to a higher minimum wage. The higher minimum wage hypothetical citizen had extra lifetime income of $\$ 170,436$ when compared with the lower minimum wage hypothetical citizen. Assuming that this extra amount will become disposable income, and using a Harmonized Sales Tax (HST) rate of 13 percent, the government is expected to receive an extra $\$ 22,156$ in sales taxes per citizen due to a one-dollar increase in minimum wage. This difference in taxes could lead one to conclude that adjusting the minimum wage is a potential way to generate revenues to 
facilitate the full funding of higher education by using the extra generated taxes. Indexing the minimum wage and linking the increase in minimum wage to the CPI could undertake this policy.

\section{RECOMMENDATIONS AND CONCLUSION}

This study suggests that the following goals could be achieved by adopting a minimum wage indexing policy:

1. Higher income tax revenues will be generated by the government to fund public programs like higher education.

2. Higher income for the most vulnerable workers (those who depend on minimum wage).

3. A reduction in the breakeven gap for government with respect to its minimum wage citizens.

4. A reduced dependence on the welfare system by making work more attractive due to higher wages.

5. Increased economic activity due to the expected higher disposable income of minimum wage workers.

A one-dollar difference in the minimum wage could be powerful enough to pay for the full cost of human capital development through higher education. A slight adjustment to the hourly wage due to a slightly higher level of training would translate into a lifetime national average of $\$ 48,301$ more in income taxes per person. These extra lifetime taxes can positively affect governments' budgets by increasing revenues in one side of the budget, and by reducing expenditures on government subsidies in the other side due to the reduction in the breakeven age of the two compared hypothetical citizens by 4.6 years.

\section{AUTHOR INFORMATION}

Mahmoud Yousef Askari is an assistant professor of management in the Department of Management \& MIS, College of Business Administration, Al Ain University of Science and Technology, P O Box 64141, Al Ain, UAE. Email:mahmoud.askari@aau.ac.ae

\section{REFERENCES}

Askari, M. (2014). A partnership approach to public finance for higher education funding in Canada (Doctoral Dissertation). Retrieved from Dissertations and Theses database, University of Calgary http://hdl.handle.net/11023/1383.

AUCC (2008). Trends in higher education, volume 3: Finance. Ottawa: AUCC

http://www.aucc.ca/wp-content/uploads/2011/06/trends_2008_vol3_e.pdf.

Campolieti, M., Fang, T., \& Gunderson, M. (2005). How minimum wages affect schooling-employment outcomes in canada, 1993-1999. Journal of Labor Research, 26(3), 533-545. Retrieved from doi:http://dx.doi.org/10.1007/s12122-005-1019-z

Campolieti, M., Gunderson, M., \& Lee, B. (2012). The (non) impact of minimum wages on poverty: Regression and simulation evidence for canada. Journal of Labor Research, 33(3), 287-302. Retrieved from doi:http://dx.doi.org/10.1007/s12122-012-9139-8

Card, D., \& Krueger, A. B. (1995). Time-series minimum-wage studies: A meta-analysis. The American Economic Review, 85(2), 238. Retrieved from http://search.proquest.com/docview/233026818?accountid=130846

Dickson, V., \& Myatt, T. (2002). The determinants of provincial minimum wages in canada. Journal of Labor Research, 23(1), 57-67. Retrieved from http://search.proquest.com/docview/214017974?accountid=130846

Edwards, P., \& Gilman, M. (1999). Pay equity and the national minimum wage: What can theories tell us? Human Resource Management Journal, 9(1), 20-38. Retrieved from http://search.proquest.com/docview/199448047?accountid=130846

Even, W. E., \& Macpherson, D. A. (1996). Consequences of minimum wage indexing. Contemporary Economic Policy, 14(4), 67-77. Retrieved from http://search.proquest.com/docview/274335929?accountid=130846

Friedman, M. and Friedman R. (1980). Free to Choose: A Personal Statement. London:

Secker and Warburg. 
Hauptman, A. M., \& Nolan, P. (2011). Assessing the effects of four budget-balancing strategies in higher education. Higher Education Management And Policy, 23(1).

Metcalfe, A. (2010). Revisiting academic capitalism in Canada: No longer the exception. Journal Of Higher Education, 81(4), 489-514.

Partridge, M. D., \& Partridge, J. S. (1999). Do minimum wage hikes raise US long term unemployment? evidence using state minimum wage rates. Regional Studies, 33(8), 713-726. Retrieved from http://search.proquest.com/docview/208878912?accountid=130846

Sun, F., \& Barrientos, A. (2009). The equity challenge in China's higher education finance policy. Higher Education Policy, 22(2), 191-207.

Woodhall, M. (2007). Funding higher education: The contribution of economic thinking to debate and policy development. Working Paper No. 8. Washington: The World Bank: http://siteresources.worldbank.org/EDUCATION/Resources/278200-1099079877269/5476641099079956815/Funding HigherEd wps8.pdf. 


\section{APPENDIX A}

Government Transfer Payments by Category

Annual government transfer payments to persons (dollars x 1,000,00)

\begin{tabular}{|c|c|c|c|c|c|c|}
\hline Geography & Government transfer payments to persons & 2005 & 2006 & 2007 & 2008 & 2009 \\
\hline Canada & Total government transfer payments & 136247 & 145754 & 154609 & 165101 & 176630 \\
\hline Canada & Total federal & 67903 & 70547 & 76578 & 81119 & 88051 \\
\hline Canada & Family and youth allowances & 165 & 187 & 205 & 210 & 211 \\
\hline Canada & Child tax benefit or credit & 9174 & 9470 & 9495 & 9468 & 6716 \\
\hline Canada & Universal child care benefit & & 1175 & 2451 & 2518 & 2590 \\
\hline Canada & Pensions, World Wars I and II & 1584 & 1693 & 1694 & 1696 & 1686 \\
\hline Canada & War veterans"' allowances & 289 & 331 & 464 & 588 & 639 \\
\hline Canada & Grants to aboriginal persons and organizations & 5752 & 5823 & 6179 & 7534 & 6532 \\
\hline Canada & Goods and Services Tax credit & 3472 & 3566 & 3599 & 3692 & 3742 \\
\hline Canada & Employment insurance benefits & 12937 & 12498 & 12561 & 13275 & 18755 \\
\hline Canada & Old age security payments & 29085 & 30468 & 31929 & 33538 & 34973 \\
\hline Canada & Scholarships and research grants & 789 & 858 & 883 & 922 & 880 \\
\hline Canada & Miscellaneous and other transfers & 4656 & 4478 & 7118 & 7678 & 8127 \\
\hline Canada & Total provincial & 33297 & 38570 & 39446 & 42922 & 45030 \\
\hline Canada & Social Insurance benefits, workers' compensation & 5229 & 5316 & 5500 & 5797 & 5970 \\
\hline Canada & Social insurance benefits, other & & 830 & 1452 & 1561 & 1649 \\
\hline Canada & Grants to benevolent associations & 9581 & 10593 & 11280 & 12153 & 12877 \\
\hline Canada & Social assistance, income maintenance & 6918 & 7123 & 7480 & 7784 & 8232 \\
\hline Canada & Social assistance, other & 3445 & 3791 & 3989 & 4354 & 4986 \\
\hline Canada & Miscellaneous transfers & 8124 & 10917 & 9745 & 11273 & 11316 \\
\hline Canada & Total local & 3026 & 2976 & 3305 & 3827 & 7268 \\
\hline Canada & Canada Pension Plan (CPP) & 24225 & 25417 & 26624 & 28089 & 29611 \\
\hline Canada & Quebec Pension Plan (QPP) & 7796 & 8244 & 8656 & 9144 & 9670 \\
\hline
\end{tabular}

Source: Statistics Canada. Table 384-0009 - Government transfer payments to person, provincial economic accounts, annual (dollars) (accessed: May 21, 2013).

\section{APPENDIX B}

Federal Income Tax Rates in Canada (2012)

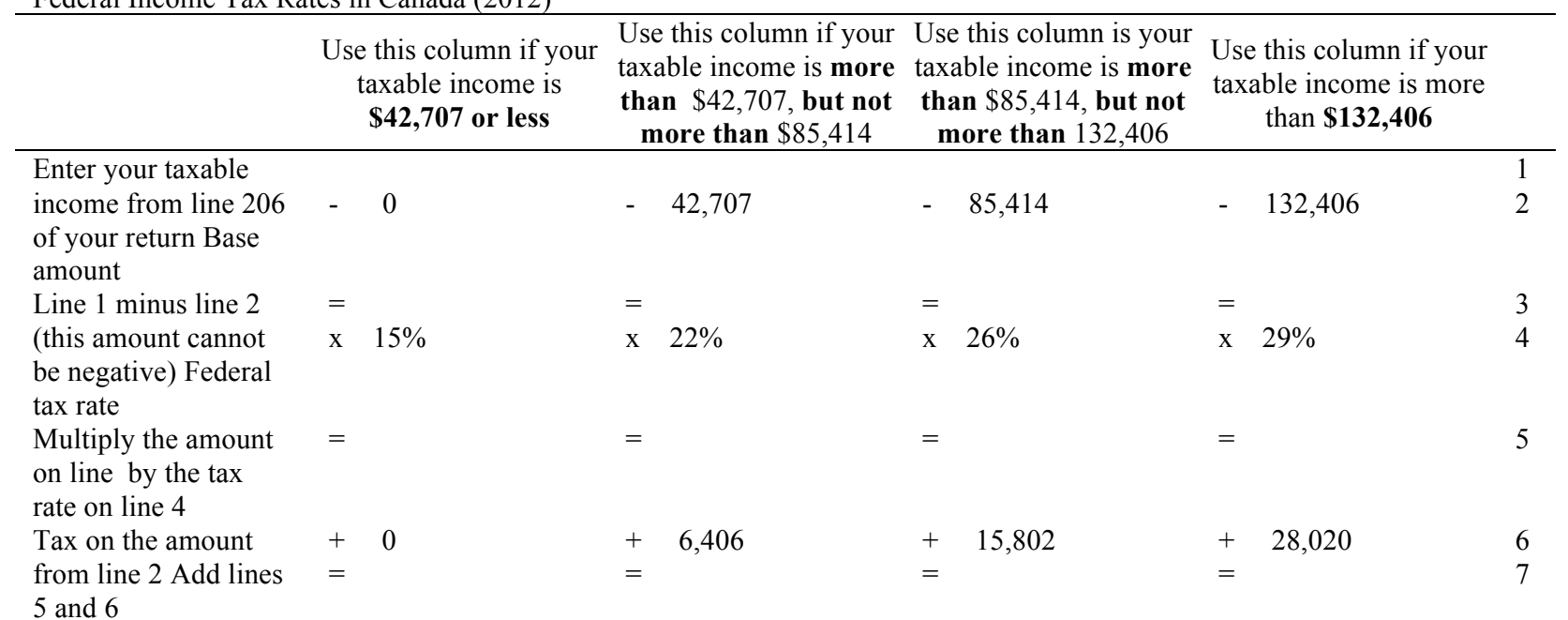

Note: under the current tax on income method, tax for all provinces (except Quebec) and territories is calculated the same way as federal tax. Form 728 is used to calculate this provincial or territorial tax. Provincial or territorial specific non-refundable tax credits are also calculate on Form 428. For complete details, see the provincial or territorial information and dorms in your 2012 tax package.

Source: Canada Revenue Service

Date modified: 1/03/13 


\section{APPENDIX C}

Provincial Income Tax Rates (2012)

Provincial/territorial tax rates (combined chart)

Provinces/Territories $\quad$ Rates(s)

Newfoundland and Labrador

$7.7 \%$ on the first $\$ 32,893$ of taxable income, +

$12.5 \%$ on the next $\$ 32,892,+$

Nova Scotia

$13.3 \%$ on the amount over $\$ 65,785$

$8.79 \%$ on the first $\$ 29,590$ of taxable income, +

$14.95 \%$ on the next $\$ 29,590,+$

$16.67 \%$ on the next $\$ 33,820,+$

$17.5 \%$ on the next $\$ 57,000,+$

New Brunswick

$21 \%$ on the amount over $\% 150,00$

$9.1 \%$ on the first $\$ 38,190$ of taxable income, +

$12.1 \%$ on the next $\$ 38,190$, +

$12.4 \%$ on the next $\$ 47,798,+$

$14.3 \%$ on the amount over $\$ 124,178$

Qubec

Ontario

See income tax rates (Revenue Quebec Web site)

$5.05 \%$ on the first $\$ 39,020$ of taxable income, +

$11.16 \%$ on the next $\$ 421,957,+$

$12.4 \%$ on the next $\$ 47,798,+$

$12.16 \%$ on the amount over $\$ 500,000$

Manitoba

$10.8 \%$ on the first $\$ 31,000$ of taxable income, +

$12.75 \%$ on the next $\$ 36,000,+$

$17.4 \%$ on the amount over $\$ 67,000$

Sakatchewan

$11 \%$ on the first $\$ 42,065$ of taxable income, +

$13 \%$ on the next $\$ 78,120,+$

$15 \%$ on the next $\$ 120,185,+$

Alberta

$10 \%$ of taxable income

British Columbia

$5.06 \%$ on the first $\$ 37,013$ of taxable income, +

$7.7 \%$ on the next $\$ 37,015,+$

$10.5 \%$ on the next $\$ 10,965,+$

$12.29 \%$ on the next $\$ 18,212$, +

$14.7 \%$ on the amount over $\$ 103,205$

Yukon

$7.04 \%$ on the first $\$ 42,707$ of taxable income, +

$9.68 \%$ on the next $\$ 72,707,+$

$11.44 \%$ on the next $\$ 46,992,+$

$12.76 \%$ on the amount over $\$ 132,406$

Northwest Territores

$5.9 \%$ on the first $\$ 38,679$ of taxable income, +

$8.6 \%$ on the next $\$ 38,681,+$

$12.2 \%$ on the next $\$ 48,411,+$

$14.05 \%$ on the amount over $\$ 125,771$

Nunavut

$4 \%$ on the first $\$ 40,721$ of taxable income, +

$7 \%$ on the next $\$ 40,721,+$

$9 \%$ on the amount over $\$ 50,964$

Source: Canada Revenue Agency

Date modified: 0/03/13 